\title{
Vortex Dynamics in Trabeculated Embryonic Ventricles
}

\author{
Nicholas A. Battista ${ }^{1, *(1)}$, Dylan R. Douglas ${ }^{2,3}$, Andrea N. Lane ${ }^{4}$, Leigh Ann Samsa ${ }^{5}$, \\ Jiandong Liu ${ }^{6,7}$ (D) and Laura A. Miller ${ }^{2,3}$ \\ 1 Department of Mathematics and Statistics, 2000 Pennington Road, The College of New Jersey, \\ Ewing Township, NJ 08628, USA \\ 2 Department of Biology, 3280, University of North Carolina, Chapel Hill, NC 27599, USA; \\ ddray@live.unc.edu (D.R.D.); lam9@unc.edu (L.A.M.) \\ 3 Department of Mathematics, CB 3250, University of North Carolina, Chapel Hill, NC 27599, USA \\ 4 Department of Biostatistics and Bioinformatics, Emory University, Atlanta, GA 30307, USA; anlane@me.com \\ 5 Department of Cell Biology and Physiology, University of North Carolina, Chapel Hill, NC 27599, USA; \\ lasamsa@ncsu.edu \\ 6 McAllister Heart Institute, UNC School of Medicine, University of North Carolina, Chapel Hill, NC 27599, \\ USA; jiandong_liu@med.unc.edu \\ 7 Department of Pathology and Laboratory Medicine, University of North Carolina, \\ Chapel Hill, NC 27599, USA \\ * Correspondence: battistn@tcnj.edu; Tel.: +1-716-517-6561
}

Received: 17 September 2018; Accepted: 18 January 2019; Published: 22 January 2019

\begin{abstract}
Proper heart morphogenesis requires a delicate balance between hemodynamic forces, myocardial activity, morphogen gradients, and epigenetic signaling, all of which are coupled with genetic regulatory networks. Recently both in vivo and in silico studies have tried to better understand hemodynamics at varying stages of veretebrate cardiogenesis. In particular, the intracardial hemodynamics during the onset of trabeculation is notably complex-the inertial and viscous fluid forces are approximately equal at this stage and small perturbations in morphology, scale, and steadiness of the flow can lead to significant changes in bulk flow structures, shear stress distributions, and chemical morphogen gradients. The immersed boundary method was used to numerically simulate fluid flow through simplified two-dimensional and stationary trabeculated ventricles of 72, 80, and $120 \mathrm{~h}$ post fertilization wild type zebrafish embryos and ErbB2-inhibited embryos at seven days post fertilization. A 2D idealized trabeculated ventricular model was also used to map the bifurcations in flow structure that occur as a result of the unsteadiness of flow, trabeculae height, and fluid scale $(R e)$. Vortex formation occurred in intertrabecular regions for biologically relevant parameter spaces, wherein flow velocities increased. This indicates that trabecular morphology may alter intracardial flow patterns and hence ventricular shear stresses and morphogen gradients. A potential implication of this work is that the onset of vortical (disturbed) flows can upregulate Notch1 expression in endothelial cells in vivo and hence impacts chamber morphogenesis, valvulogenesis, and the formation of the trabeculae themselves. Our results also highlight the sensitivity of cardiac flow patterns to changes in morphology and blood rheology, motivating efforts to obtain spatially and temporally resolved chamber geometries and kinematics as well as the careful measurement of the embryonic blood rheology. The results also suggest that there may be significant changes in shear signalling due to morphological and mechanical variation across individuals and species.
\end{abstract}

Keywords: trabeculae; heart development; cardiac fluid dynamics; cavity flow; immersed boundary method; fluid dynamics 


\section{Introduction}

Since the developing heart and embryo continue to function for some time in the absence of erythrocytes, it appears that the function of the early embryonic heart is not for the purpose of nutrient transport [1,2]. Instead, recent work suggests that the heart's function is to aid in its own growth [1,3-5]. Two important roles for intracardial fluid dynamics in terms of proper cardiogenesis are to exert hemodynamic forces onto the ventricular lining and to advect morphogens $[2,6]$. These two fluid effects help regulate and drive organogenesis in developing embryos. Both shear stress and pressure may be key components to activating developmental regulatory networks by acting on cardiac cells [7] through a process called mechanotransduction. In this case, mechanical stimuli are transmitted by intracellular signalling pathways to the interior of the cell. Moreover, increased receptor-ligand bond formation may appear near the endothelial lining in regions of higher vorticity [8], which gives rise to greater mixing of chemical morphogens. These chemicals may act as epigenetic signals, which are then advected throughout the chamber $[9,10]$. It is clear that irregular hemodynamics leads to cardiomyopathies or embryonic death [3,11-14].

The first heart beat occurs around $\sim 1 \mathrm{dpf}$ when its morphology resembles a simple valveless tube. It is composed of both an outer myocardial and endocardial layer of cells. Around $1.5 \mathrm{dpf}$ cardiac looping and chamber ballooning begins, where the heart drastically begins to reshape itself into a multi-chambered pumping system. Two chambers can be distinctly seen by $2 \mathrm{dpf}$, while endocardial cushions, the precursors to valve leaflets, are in the process of forming. Trabeculae, irregular muscular protrusions that expand from the inner surface of the ventricle, begin to form around $3 \mathrm{dpf}$. These stages are illustrated in Figure 1.

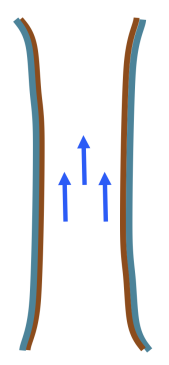

$1 d p f$

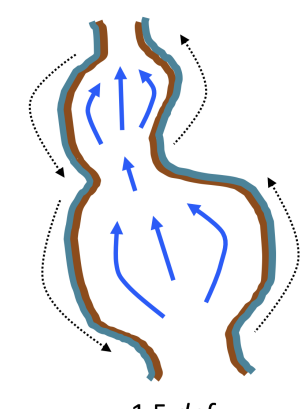

$1.5 d p f$

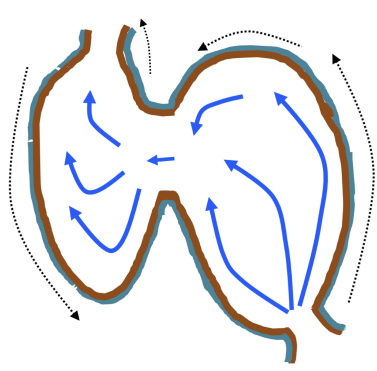

$2 d p f$

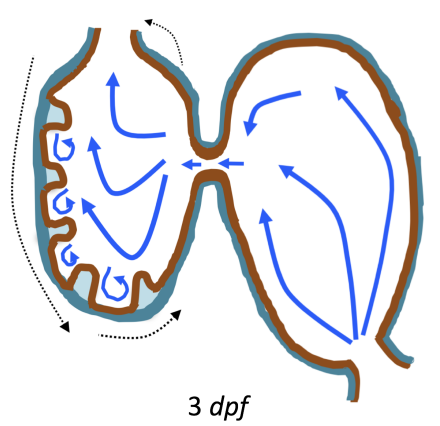

Reconfiguration stresses

Figure 1. Illustrations of significant stages of zebrafish heart development. At $1 \mathrm{dpf}$ the heart begins pumping as a valveless heart tube, at $1.5 \mathrm{dpf}$ cardiac looping and chamber ballooning begins, at $2 \mathrm{dpf}$ the heart is a growing two-chambered pumping system with endocardial cushions forming, and at $3 \mathrm{dpf}$ ventricular trabeculation occurs. The blue arrows indicate blood flow while the black dotted arrows represent mechanical stresses re-configuring the heart.

Prior to trabeculation, the endocardial ventricular cells are smooth and polygonal in shape. During the onset of trabeculation, several endocardial cells become elongated and some extend cellular projections. Moreover, these cells appear slightly more depressed than the surrounding endocardial cells. The depressions progressively become deeper and wider such that the endocardial cells invaginate the cardiac jelly and extend toward the basal surface of the myocardium. Eventually, myocardial cells separate due to the potent endocardial cell invasion, and definitive trabeculae are formed [15]. Hence, trabeculae are composed of both myocardial and endocardium components.

Proper trabeculation requires well-coordinated cardiac contraction [16] and is particularly sensitive to local changes in the fluid environment [17]. It is thought that the trabeculae may serve as mechanotranductive structures and alter intracardial flows in a way that regulates shear stress and mixing near the endocardium [18-20]. Even if subtle trabeculation irregularities were 
masked, cardiac defects would magnify over time because of their effect on morphogenetic processes. For example, zebrafish embryos designed to lack normal trabeculation (ErbB2-inhibited) displayed severe cardiovascular defects including bradycardia (decreased heart rate), decreased fractional shortening, and impaired cardiac conduction [21]. Lack of trabeculae or irregularly formed trabeculae will cause irregular patterns of shear stresses. This in turn can cause dysfunctional myocardial activation patterns that are known to cause arrhythmias, abnormal fractional shortening, and even ventricullar fibrillation [22].

During the onset of trabeculation, the underlying fluid dynamics are particularly interesting due to the balance of inertial and viscous forces. The Reynolds number, $R e$, is a dimensionless number that describes the ratio of inertial to viscous forces in the fluid. It is given by $\operatorname{Re}=(\rho U L) / \mu$, where $\mu$ and $\rho$ are the dynamic viscosity and density of embryonic blood, respectively, and $L$ and $U$ are characteristic length and velocity scales. The characteristic velocity is often chosen as the average or peak flow rate, while $L$ is often selected as the diameter of the chamber or vessel. When trabeculation begins as cardiac looping and ballooning progress, the $R e$ is approximately 1 . At this fluid scale, a number of important fluid dynamic transitions can occur. One notable feature is the transition to vortical (disturbed) flow and hence changes in flow direction. This transition is sensitive to the growing complex morphology, effective viscosity of the blood, and unsteadiness of the flow.

Disturbed blood flow patterns have been observed during heart development [23]. These flow patterns generally induce lower wall shear stresses (WSS) than smoothly streaming (non-disturbed) blood flow. Both contribute to remodelling and endothelial cell activation in different ways [24,25]. It has been shown that disturbed (vortical) flow patterns upregulate the expression of certain genes, such as Notch1, in endothelial cells during development [26]. As the heart undergoes dramatic morphological transformations, transitions to vortical and disturbed flow patterns may help guide morphogenesis through changing patterns in WSS or through other mechanotransductive mechanisms such as flow sensing through primary cilia [27-29]. Note that intracardial flows are both temporally and spatially varying such that the distribution of WSS is not uniform along the endothelium $[19,20]$. Hence, mechanotransducers will exhibit different responses, leading to differentiated cellular behavior [30]. Furthermore, as the heart grows, blood flow also increases [31]. The formation of complex structures along the ventricle, like trabeculae, may provide regions where disturbed flow develops which could lead to higher kinetic energy dissipation. This energy dissipation may facilitate proper ventricle contractile function and trabecular organization [20].

Due to the complexity of the cardiogenesis and the challenges of measuring flow patterns precisely, computational fluid dynamics (CFD) has become a premier tool for resolving the flow in embryonic hearts [19,20,32-41]. For example, Liu et al. [34] simulated flow through a three-dimensional model of a chick embryonic heart during stage $\mathrm{HH} 21$ (after about 3.5 days of incubation) at a maximum Re of about 6.9. They found that vortices formed during the ejection phase near the inner curvature of the outflow tract. In 2013, Lee et al. [38] performed 2D simulations of the developing zebrafish heart with moving cardiac walls. They found that unsteady vortices develop during atrial relaxation at $20-30 \mathrm{hpf}$ and in both the atrium and ventricle at 110-120 hpf.

More recently, Vedula et al. [19] and Lee et al. [20] used light-sheet fluorescent microscopy and reconstructed a $4 D$ moving ventricle on which they based their CFD model. They were able to quantify spatially- and temporally-varying WSS along trabceular ridges (trabeculae "heads") and groves ("intertrabecular regions"). In particular, Lee et al. discovered that pulsatile shear-stresses developed along the ridges at $3 \mathrm{dpf}$ in wildtype (WT) zebrafish embryos, while oscillatory shear-stresses (OSS) developed in the groves around $4 \mathrm{dpf}$ [20]. Around $4 \mathrm{dpf}$, vortical flow patterns may be present within the intertrabecular spaces. Moreover, OSS were found to be substantially less at the trabecular heads, suggesting that OSS may be a possible regulatory control during cardiogenesis $[19,20]$. They also investigated differences in WSS between wildtype and mutant zebrafish hearts. The mutants they considered were ErbB2-inhibited zebrafish (suppresses trabeculation), gata1a morpholinos (lowers blood viscosity), and wea mutants (lower cardiac contractility). They found that total WSS 
was comparable in the WT and ErbB2-inhibited zebrafish; however, the gata1a morpholinos and wea mutants expressed significantly less total WSS. Another study, Battista et al. [40], found that trabeculae morphology has a significant effect on intertrabecular vortex formation, as does the presence of hematocrit and fluid scale. However, their study did not include an analysis of WSS, but instead referred to the tangential, normal, and total force magnitudes as potential proxies for WSS, although they observed similar trends to the spatially-averaged WSS over the course of a heart cycle.

The numerical work described above and in vivo measurements of blood flow in embryonic hearts [3,42] supports that vortex formation is sensitive to changes in $R e$, morphology, and unsteadiness of the flow. Santhanakrishnan et al. [35] used a combination of CFD and flow visualization in dynamically scaled physical models to describe the fluid dynamic transitions that occur as the chambers balloon, the endocardial cushions grow, and the overall scale of the heart increases. They found that the formation of intracardial vortices depends upon the height of the endocardial cushions, the depth of the chambers, and the $R e$. Their study only considered steady flows in an idealized two-dimensional chamber geometry with smooth, stationary walls.

In this paper, we present complementary studies to both Santhanakrishnan et al. [35] and Lee et al. [20] with the goal of revealing the bifurcations in flow structures that occur as a result of the unsteadiness of the flow, trabeculae height, and Re. First, we investigate the differences in the cardiac fluid dynamics between WT and ErbB2-inhibited (namely ErbB2 ${ }^{\text {st61 }}$ and ErbB2 ${ }^{\text {st50 }}$ ) mutants, to explore how vortex formation in the intertrabecular regions is sensitive to differences in morphology. We quantify the intertrabecular flow patterns mentioned (but not shown) in Lee et al. [20]. Next, we use an idealized geometry, based upon that of Santhanakrishnan et al. [35], to systematically sweep a parameter space consisting of trabeculae size, fluid scale, and unsteady flow effects to quantify fluid dynamics transitions.

It is important to note that, in this study, we perform 2D simulations using stationary boundaries. This permits direct comparison to the steady flows moved through the simplified chambers considered by Santhanakrishnan et al. [35]. It also permits exploration of a wide parameter space that covers the range of biological diversity, including other trabeculated vertebrate embryonic hearts and trabeculated invertebrate hearts. As such, the results highlight parameter spaces where bulk flow patterns are highly sensitive to small changes in morphology, effective viscosity, and unsteadiness of the fluid. In such parameter regions, it is critical to obtain highly resolved descriptions of morphology, pumping kinematics, and rheology of the blood. The simulation results shown here are not as realistic as those presented for the zebrafish embryonic hearts simulated in 3D with moving boundaries by Lee et al. [20] and Vedula et al. [19]. Our goal, however, was to map the parameter space sensitive to small changes in a relatively simple model. We believe that the results will serve as motivation for more detailed three-dimensional studies. We also argue that the stationary boundaries and 2D approximations serve as a reasonable starting point given the relatively low Womersley number, $W o \approx 0.77$, since unsteady effects become significant for $W_{o}>1$.

\section{Methods}

Two-dimensional computational fluid dynamics (CFD) simulations were used to quantify the flow fields within a biologically-realistic and an idealized model of a trabeculated ventricle of the zebrafish embryonic heart. We will discuss the model geometry construction below and emphasize that the purpose of this study is to illustrate that flow patterns in and near trabeculated regions are particularly sensitive to small changes in morphology and scale in a biologically relevant parameter space. For a detailed discussion on the numerical method used to solve the fluid-structure problem, see Appendix A.

\subsection{Embryonic Zebrafish Model Geometry}

We estimated the structure of trabeculae from in-vivo image data taken from embryonic zebrafish for the purpose of simulating flow through realistic ventricle geometries. Five images of stained cross 
sections of both wildtype and mutant embryonic zebrafish were taken from Liu et al. [21]. The mutant and transgenic lines used were $E r b B 2^{s t 61}$ and $E r b B 2^{s t 50}$. Figures from the original paper were cropped out and manually traced by recording pixel locations along the boundary with the open-source Python package Argus [43]. Smooth-spline approximations of the trabeculated ventricle walls were generated and sampled to generate the discretized computational geometry as seen in Figure 2.

Figure 2 shows the computational geometries extracted from microscopy images [21]. Note that the images were acquired using a Nikon Te-2000u microscope (UNC-CH, Chapel Hill, NC, USA) at a rate of 250 frames per second with a high-speed CMOS camera (MiCam Ultima, SciMedia, $\mathrm{UNC}-\mathrm{CH}$ ). The geometries chosen for this study were of wild-type embryos at 3 and $5 \mathrm{dpf}$ and an ErbB2 inhibited embryo at $7 \mathrm{dpf}$. Note that, at $5 \mathrm{dpf}$, ErbB2-inhibited embryos show little to no signs of trabeculation [21]; however, at $7 \mathrm{dpf}$, there is the onset of trabeculation, as shown above. Furthermore, we considered other wild-type embryos at $80 \mathrm{hpf}$ and $5 \mathrm{dpf}$ to span biological variation; see Figure A1 in Appendix B for their geometries. Note that we distinguish between both $5 \mathrm{dpf}$ embryos by giving them an (I) or (II) designation.

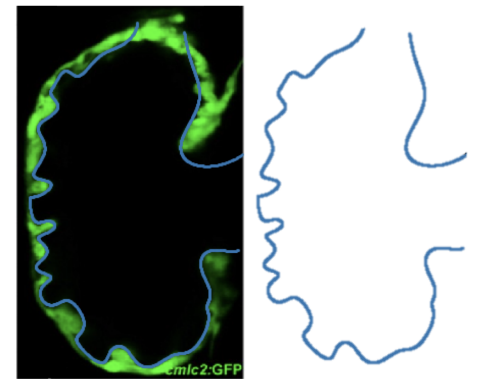

$3 d p f$ wild-type

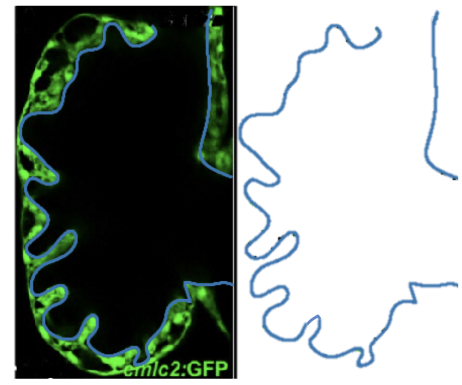

$5 d p f$ wild-type (I)
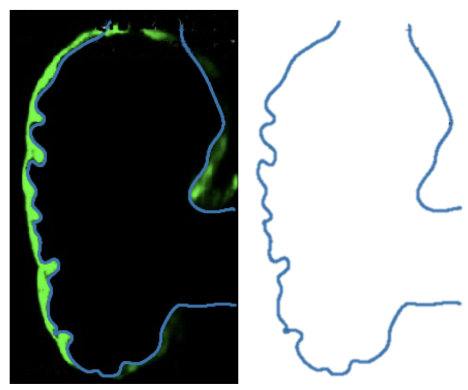

7 dpf ErbB2 Inhibited

Figure 2. The extracted geometries from trabeculated ventricles at different stages of development from Liu et al. [21]. There are two wild-type (WT) zebrafish cases shown ( $3 \mathrm{dpf}$ and $5 \mathrm{dpf}(\mathrm{I})$ ) as well as the geometry taken from an ErbB2-inhibited zebrafish at $7 \mathrm{dpf}$.

To ensure the simulations were conducted at the appropriate fluid scale, we computed the biologically relevant $R e$ to encapsulate the correct fluid dynamic regime. For the Re within the ventricle of a $4 \mathrm{dpf}$ wild type zebrafish, the characteristic velocity, $V_{z f}$, was taken as the average of the minimum and maximum velocity measured in vivo, and the characteristic length, $L_{z f}$, was taken as the width of the ventricle from Figure 3a. The Re was then calculated as

$$
\operatorname{Re}=\frac{\rho_{z f} L_{z f} V_{z f}}{\mu_{z f}}=1.07,
$$

where $V_{z f}=0.75 \mathrm{~cm} / \mathrm{s}$ [3], $\rho_{z f}=1025 \mathrm{~kg} / \mathrm{m}^{3}$ [5], $\mu_{z f}=0.0015 \mathrm{~kg} /(\mathrm{ms})$ [44], and $L_{z f}=208 \mu \mathrm{m}$. The characteristic frequency was chosen as $f=3.95$ beats/s [18]. The dimensionless frequency may then be calculated as

$$
\tilde{f}=\frac{L_{z f}}{V_{z f}} f_{z f}=0.11
$$

The simulations using the biologically realistic geometry were performed at $R e \sim 1$. The length scale was taken directly from the images of the embryos and the velocity, frequency, and kinematic viscosity (the ratio of the fluid's dynamic viscosity to density) were taken from the literature as described above.

Figure 4 shows how the geometric parameters were measured from the reconstructed geometries. Note that, although there is geometric variation between each case, the parameters are labeled consistently. We do not, however, vary the $R e$ for the simulations that use the realistic geometry. Note that we do vary $R e$ for the idealized trabeculated ventricle geometry described in Section 2.2. 


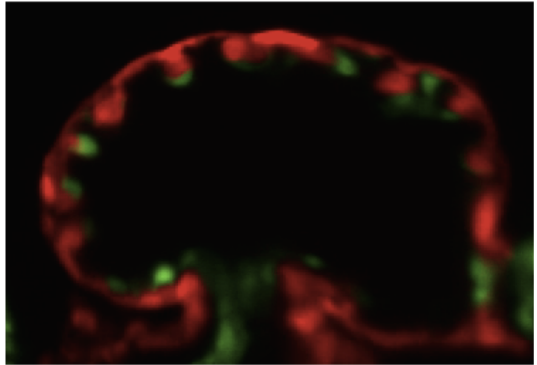

(a)

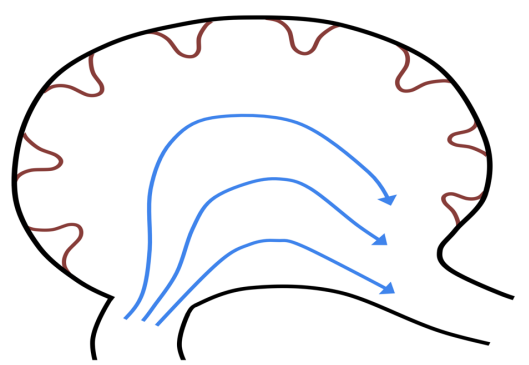

(b)

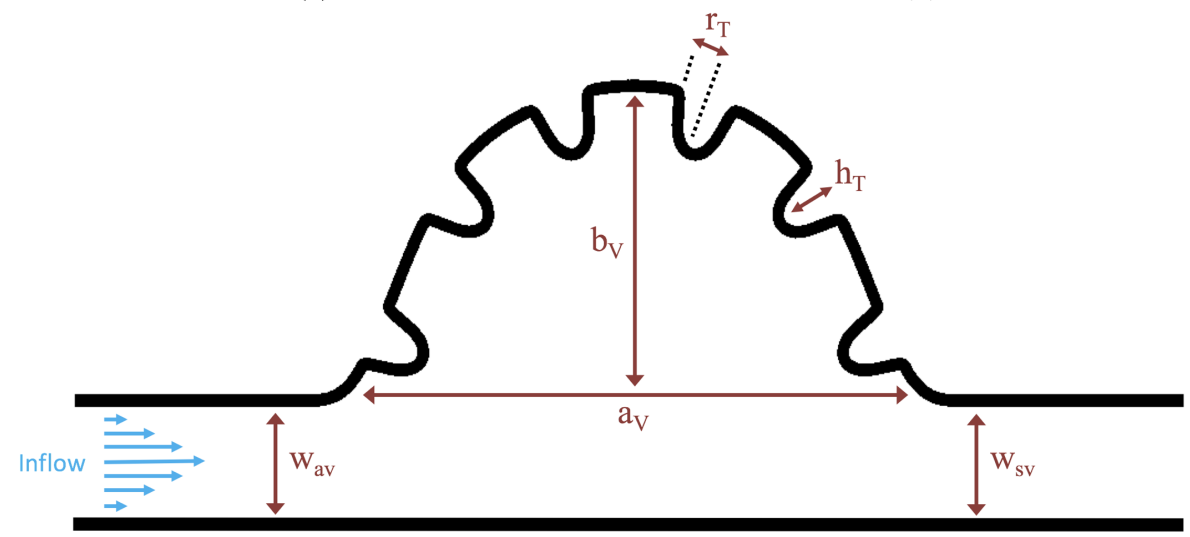

(c)

Figure 3. (a) a microscopy image of an embryonic zebrafish's trabeculated ventricle at $4 \mathrm{dpf}$ from Liu et al. [21]. This snapshot was taken immediately before the systolic phase. The protrusions into the ventricle are trabeculae. The image was taken from $\mathrm{Tg}(\mathrm{cmlc2}$ :dsRed)s879; $\mathrm{Tg}(\mathrm{flk} 1$ :mcherry)s843 embryos expressing fluorescent proteins that label the myocardium and endocardium, respectively [21]; (b) simplified diagram showing the basic idea behind our idealized geometry. Blood flows from the atrio-ventricular canal into the ventricle and then proceeds into the bulbus arteriosus; (c) further idealization of the computational model which is now flattened. The geometric parameters are as follows: $a_{V}$ and $b_{V}$ are the semi-major and semi-minor axis of the elliptical chamber, $h_{T}$ and $r_{T}$ are the height and radii of the trabeculae, and $w_{A V}$ and $w_{S V}$ are the widths of the atrioventricular (AV) canal and sinus venosus, respectively.

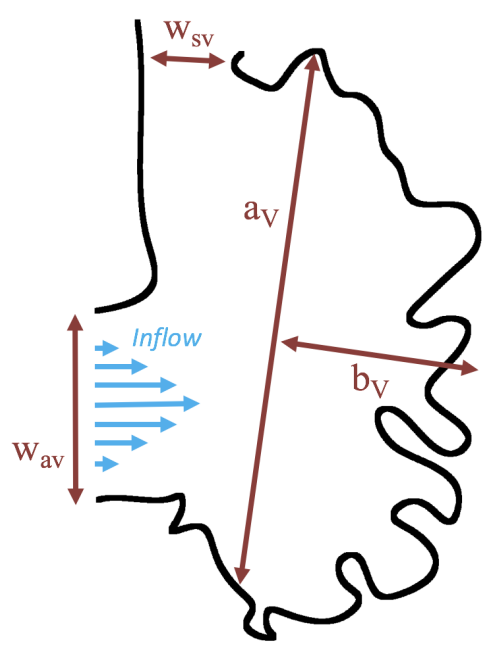

Figure 4. Schematic diagram showing the parameters for the biologically realistic simulations. Note that $a_{V}$ is the characteristic length for selecting the correct $R e$. 


\subsection{Idealized Model Geometry}

A simplified two-dimensional geometry of a $4 \mathrm{dpf}$ zebrafish's trabeculated ventricle was constructed using Figure 3a, from Liu et al. [21]. The ventricle was idealized as a half ellipse, with semi-major axis $a_{V}$ and semi-minor axis $b_{V}$. It is tangentially laid within a channel, which emulates a cavity-flow geometry. The channel models the atrioventricular canal (AV canal), with width $w_{A V}$ set equal to the width of the sinus venosus (SV), $w_{S V}$. Six equally-spaced trabeculae were aligned within the ventricle. The idealized model geometry is illustrated in Figure 3c. Note that the aspect ratio of the chamber was selected for direct comparison with Santhanakrishnan et al. [35].

The idealized trabeculae geometry was modeled using the following perturbed Gaussian-like function,

$$
\operatorname{Tr} a b(x)=h_{T}\left(1-\left(\frac{x}{r_{T}}\right)^{2}\right) e^{-\left(\frac{x}{0.7 r_{T}}\right)^{8}},
$$

where $r_{T}$ and $h_{T}$ are the radii and height of the trabeculae, respectively. The full idealized geometry can be seen in Figure 3c.

The geometric model parameters are given in Table 1. All parameters were scaled from measurements taken from Figure 3a. The parameters describing the ventricle were held constant and are given as the chamber height, $b_{V}$, chamber width, $a_{V}$, and width of the AV canal and SV, $w_{A V}$ and $w_{S V}$ respectively. Note that the radii of the trabeculae, $r_{T}$, was held constant in all numerical simulations, while the height of the trabeculae, $h_{T}$, was varied.

Table 1. Table of geometric parameters used in the idealized numerical model. The height of trabeculae, $h_{T}$, were varied for numerical experiments.

\begin{tabular}{cc}
\hline Parameter & Value \\
\hline$a_{V}$ & 1.0 \\
$b_{V}$ & 0.8 \\
$w_{A V}$ & 0.8 \\
$w_{S V}$ & 0.8 \\
$r_{T}$ & 0.10 \\
$h_{T}$ & $\{0,0.02,0.04, \ldots, 0.16\}$ \\
\hline
\end{tabular}

The temporal parameter values were chosen to keep the dimensionless frequency fixed at 0.10 for the pulsatile simulations, analogous to the model presented in Section 2.1. The Re was varied by changing the kinematic viscosity, $v=\mu / \rho$. The computational parameters are found in Table A1. For the simulations, the $R e_{s i m}$ is calculated using a characteristic length of $w_{A V}$ and a characteristic velocity set to $V_{\text {in }}$ (steady inflow) or $\frac{1}{2} V_{\text {in }}$ (pulsatile inflow). The simulations were performed for $R e_{s i m}=0.01,0.05,0.1,0.5,1,5,10,20,30,40,50,100$. The stiffness of the tether springs were chosen the minimize the deformations of the boundary to below $1 \%$ of the chamber diameter. This approach is used in the immersed boundary method to describe a nearly rigid boundary.

Note that we consider Re two orders of magnitude higher and lower than the relevant $R e \sim 1$ for a $4 \mathrm{dpf}$ zebrafish heart. The reason for this is twofold: (1) to map out the parameter space outside of the biologically relevant range and (2) to provide insight into fluid flows in other types of trabeculated hearts, such as those of some invertebrates [45,46].

\section{Results}

Below, we present the flow patterns and velocities for both biologically realistic and idealized $2 D$ models of trabeculated ventricles. The cases with biologically realistic geometries were of WT zebrafish and an ErbB2-inhibited mutant to contrast the intracardial and intertrabecular fluid dynamics of an embryonic zebrafish heart during development. The idealized geometry was used to systematically sweep over a parameter space to describe transitions in flow patterns for pulsatile flows, changes in 
trabecular height, and Reynolds Number Re. In the idealized geometry case, the Re was varied from 0.01 to 100 , and the trabecular heights were varied from zero to twice the biologically relevant height. We also quantified flow for both steady and pulsatile cases.

Streamlines were used to show the path that a passive particle would take in the flow. The streamline graphs were generated using the VisIt visualization software (v. 2.7.2, Lawrence Livermore National Laboratory, Livermore, CA, USA) [47]. The streamlines are drawn by making a contour map of the stream function, since the stream function is constant along the streamline. The stream function, $\psi(\mathbf{x}, t)$, in 2-D is defined by the following equations:

$$
\begin{gathered}
u(\mathbf{x}, t)=\frac{\partial \psi(\mathbf{x}, t)}{\partial y} \\
v(\mathbf{x}, t)=-\frac{\partial \psi(\mathbf{x}, t)}{\partial x}
\end{gathered}
$$

The streamline colors correspond to smooth, streaming flow (blue) and vortical flow (orange).

\subsection{Steady Flow through an Embryonic Zebrafish Heart}

Figure 5 gives the flow field streamlines for the steady inflow cases with biologically realistic geometries at $\operatorname{Re} \sim 1$. Parabolic inflow was used that accelerates from rest to a constant velocity, as detailed in Appendix A. The simulation data is given once the flow has reached its steady state. Results are shown for a $3 \mathrm{dpf}, 80 \mathrm{hpf}$, and two $5 \mathrm{dpf}$ wild-type embryos as well as an ErbB2-inhibited embryo at $7 \mathrm{dpf}$. In all cases, vortex formation occurred in the intertrabecular regions along the side opposite that to the sinus venosus (SV) and was within the well pronounced intertrabecular grooves. No intracardial vortices were observed and the flow smoothly steams from the atrioventricular canal to the sinus venosus.

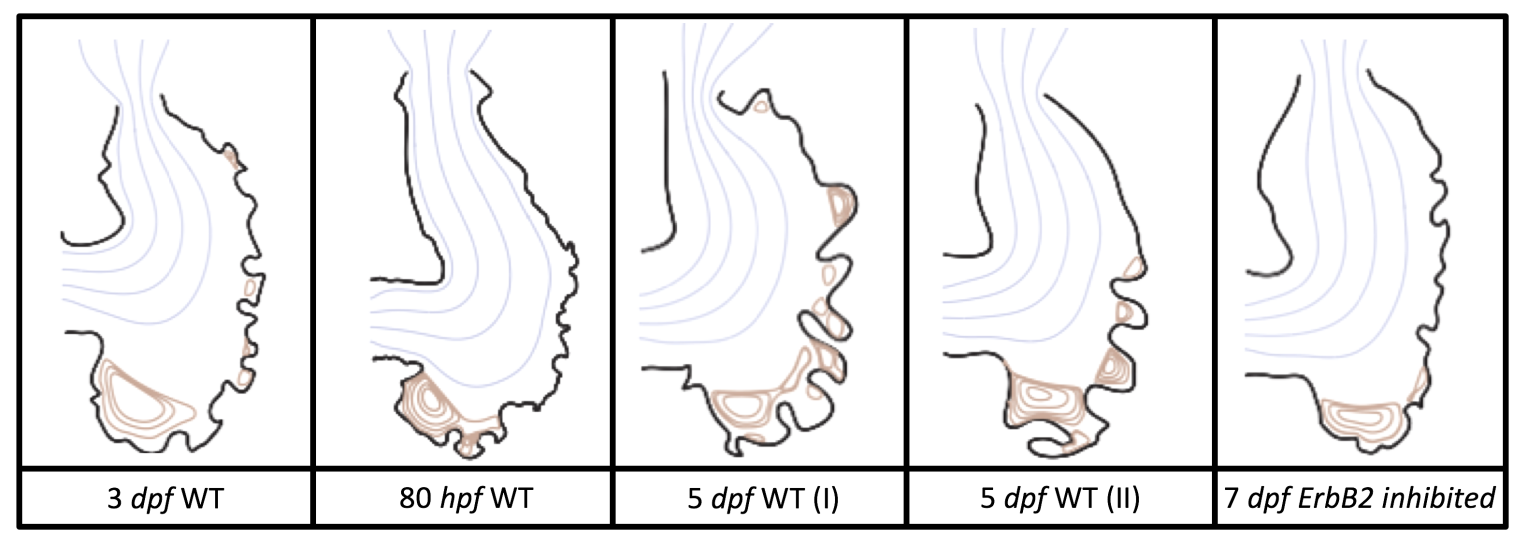

Figure 5. Streamlines showing the direction of steady flow through realistic trabeculated ventricular geometries for the biologically relevant $R e, R e=1$. The orange lines detail closed streamlines, illustrating vortical flow patterns, while blue laminar, non-vortical flow patterns.

Figure 6 illustrates that, in the regions of significant vortex formation, e.g., in the intertrabecular regions, velocities are much lower than in the intracardial region of the ventricle. In all cases, the velocities on the side opposite the SV experience much faster velocity decay towards the ventricular lining, whereas regions opposite the $\mathrm{AV}$ canal experience slower decay. Note that, in the $3 \mathrm{dpf}$ and $7 \mathrm{dpf}$ ErbB2 inhibited cases, the flow velocities along the line drawn from the AV canal to the intertrabecular region that is closest to the SV decrease in magnitude before increasing again and finally decaying to zero near the cardiac wall. This is due to the fact that the velocity is measured close to the ventricular wall that extends from the AV canal. Flow velocities measured between the trabeculae are significantly lower than those measured in within the middle of the chamber. 


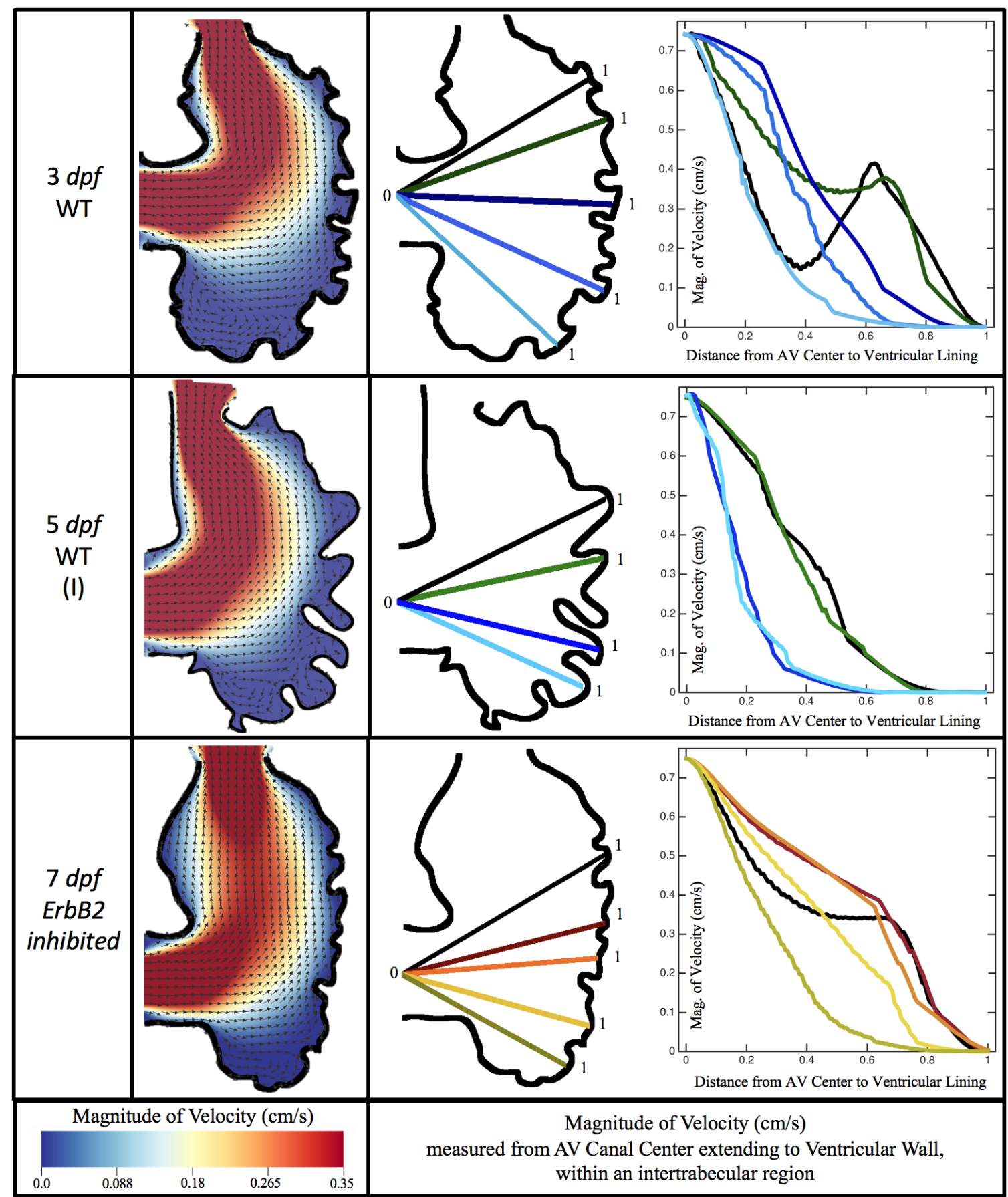

Figure 6. Simulation results for a 3 and 5 dpf WT (I) and 7 dpf ErbB2 inhibited zebrafish for Re $=1$. The magnitude of velocity $(\mathrm{cm} / \mathrm{s})$ is given by the colormap, and the arrows show the direction of flow once steady state is reached (left). The panels on the right show the magnitude of velocity along lines drawn from the center of the AV canal to the ventricular lining between the trabeculae. Note that these lines are shown in the middle panels.

\subsection{Pulsatile Flow through an Embryonic Zebrafish Heart}

In this subsection, we show the results of the pulsatile inflow simulations where biologically realistic geometries are used at $R e \sim 1$ and the frequencies are varied. Temporal snapshots of the streamlines are presented in Figure 7. The pulsatile inflow condition is detailed in Appendix A. Note that the period of the pulsation cycle is $T$. At the beginning and end of each pulsatile cycle, 
the flow is near zero. During the middle of the pulsation cycle, flow is maximal. Although this model is an idealization, we consider the first $50 \% T$ as analogous to diastole and $50-100 \% T$ as systole. All data shown is taken from the last pulse cycle such that periodic steady state has been reached. The geometries simulated include a $3 \mathrm{dpf}$, a $80 \mathrm{hpf}$, and two $5 \mathrm{dpf}$ wild-type embryos as well as an ErbB2 inhibited embryo at $7 \mathrm{dpf}$.

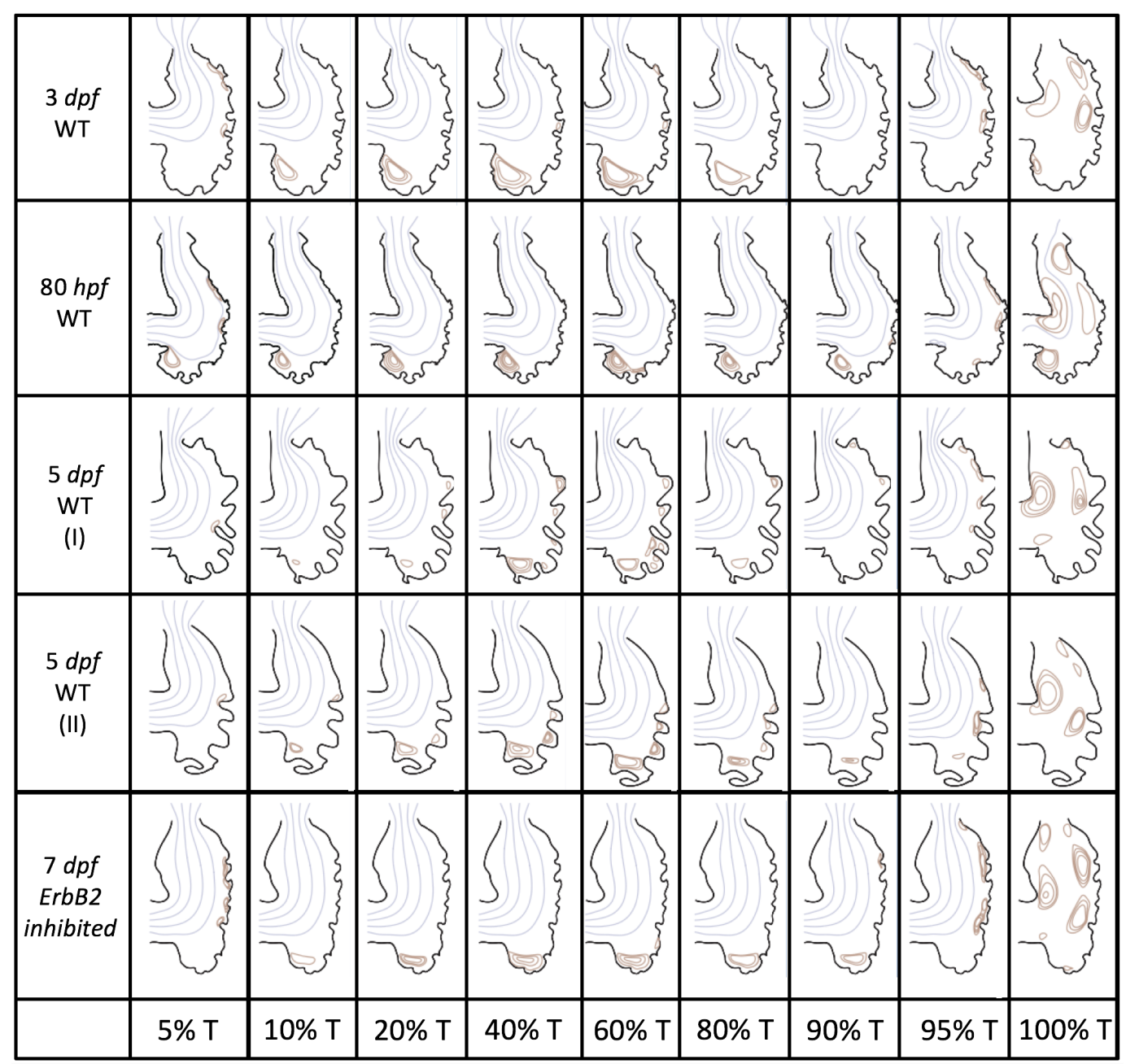

Figure 7. Temporal snapshots of the streamlines at different points during a pulsation cycle, described by a percentage of the pulsation period, $T$, within the realistic trabeculated ventricles of embryonic zebrafish from different stages of development. These simulations were run for biologically relevant $R e, R e=1$.

Vortices formed in all cases within the intertrabecular regions along the side opposite that to the sinus venosus, similar to the steady cases illustrated in Figure 5. The vortices are, however, dynamic; they change shape and size during a single pulsation cycle. Some additional vortices appear in the intertrabecular regions that were not present in the steady case. For example, consider the $80 \mathrm{hpf}$ and ErbB2-inhibited cases where vortices appear on the side opposite the AV canal. Furthermore, large intracardial vortices appear between pulsation cycles in all cases when there is minimal inflow.

Figures 8 and 9 give temporal snapshots for the $5 \mathrm{dpf}$ WT (I) and the ErbB2-inhibited cases, respectively. The colormap illustrates the magnitude of velocity $(\mathrm{cm} / \mathrm{s})$ with accompanying velocity vector fields. The velocity field is consistent with Figure 7 and shows vortex formation. These results also illustrate the spatial gradient in velocity within the intracardial to intertrabecular regions. It is clear that, although the fastest flow moves towards the middle of the chamber, the velocity significantly 
tapers off by the time it reaches the ventricular lining, the region between trabeculae on the right-hand side of the ventricular geometries in Figures 8 and 9.

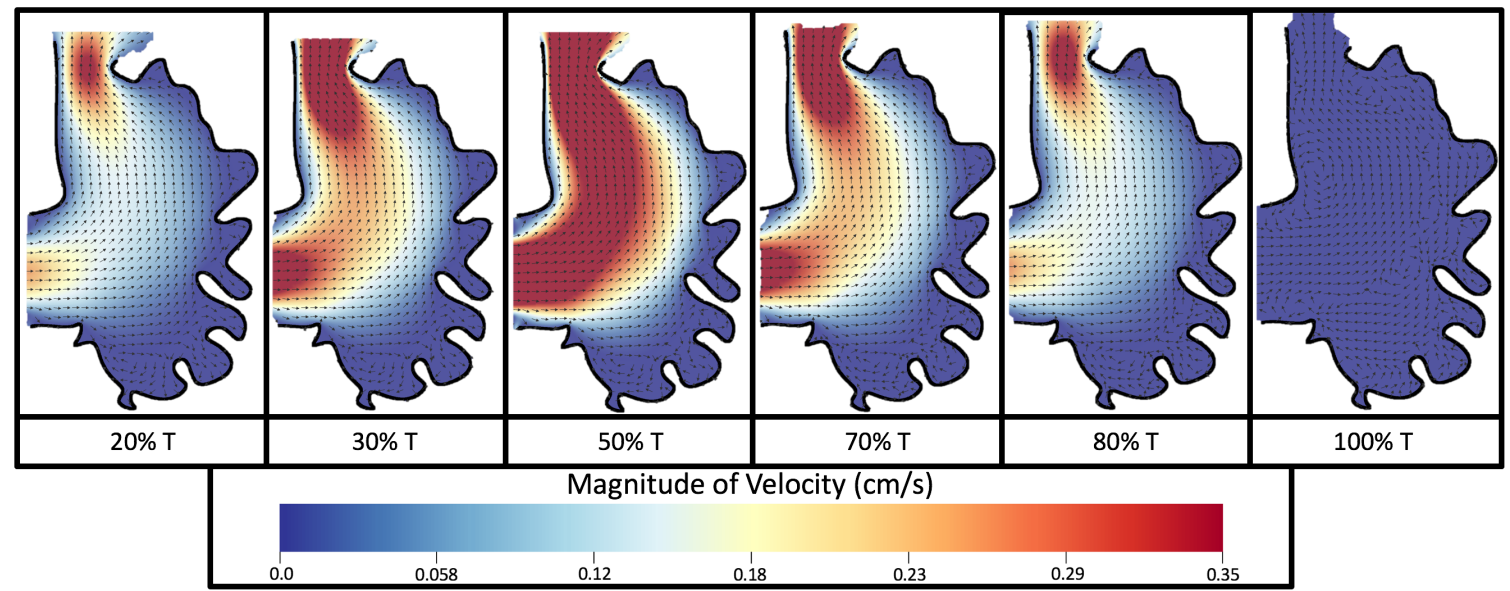

Figure 8. Temporal snapshots of the flow at different points during a pulsation cycle, described by a percentage of the pulsation period, $T$, for the $5 \mathrm{dpf}$ (I) WT ventricle at $R e=1$. The magnitude of velocity $(\mathrm{cm} / \mathrm{s})$ is given by the colormap and the direction of flow is given by the arrows.

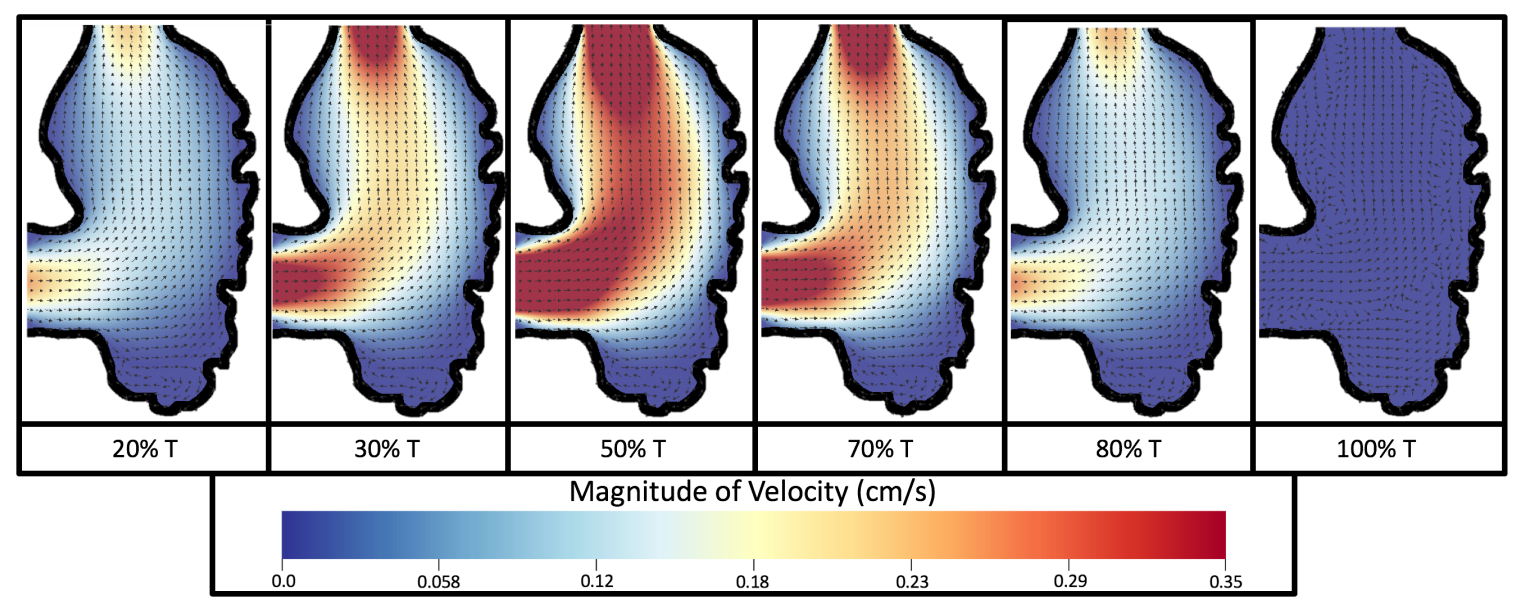

Figure 9. Temporal snapshots of the flow at different points during a pulsation cycle, described by a percentage of the pulsation period, $T$, for an ErbB2-inhibited based geometry at $7 \mathrm{dpf}$ at $\operatorname{Re}=1$. The magnitude of velocity $(\mathrm{cm} / \mathrm{s})$ is shown by the colormap along with the velocity vectors.

Figures 10-12 illustrate the magnitude of the velocity along lines drawn from the center of the AV canal to the intertrabecular regions for cases of a 3 and $5 \mathrm{dpf}$ WT (I) and $7 d p f$ ErbB2-inhibited zebrafish geometries. Appendix C presents this data in a non-logarithmic scale, to complement the data given in Figure 6 in Section 3.1. Note that the $3 \mathrm{dpf}$ WT and $7 \mathrm{dpf}$ ErbB2-inhibited zebrafish exhibit smaller trabeculae than the $5 \mathrm{dpf}$ WT (I) case.

As expected in all cases, peak flows occur within the chamber and decay as one moves toward the cardiac wall. Even in the steady inflow cases, the velocity decay is similar. The velocity decreases close to geometrically towards the intertrabecular region on the opposite side to the SV for the smaller trabeculae (3 dpf WT and $7 \mathrm{dpf}$ ErbB2-inhibited). For the $3 \mathrm{dpf}$ WT embryos in regions with pronounced trabeculation, the velocity decay does not monotonically decrease; it increases slightly within the trabeculae before decreasing to zero as one moves towards the endocardium. Similar non-monotonic behavior is seen for some intertrabecular regions farther from the SV in the $3 \mathrm{dpf}$ WT and $7 d p f$ ErbB2-inhibited embryos. We also note that the magnitude of flow decreases much more rapidly for the $5 \mathrm{dpf}$ WT (I) with pronounced trabeculation, indicating lower WSS within the intertrabecular regions. 


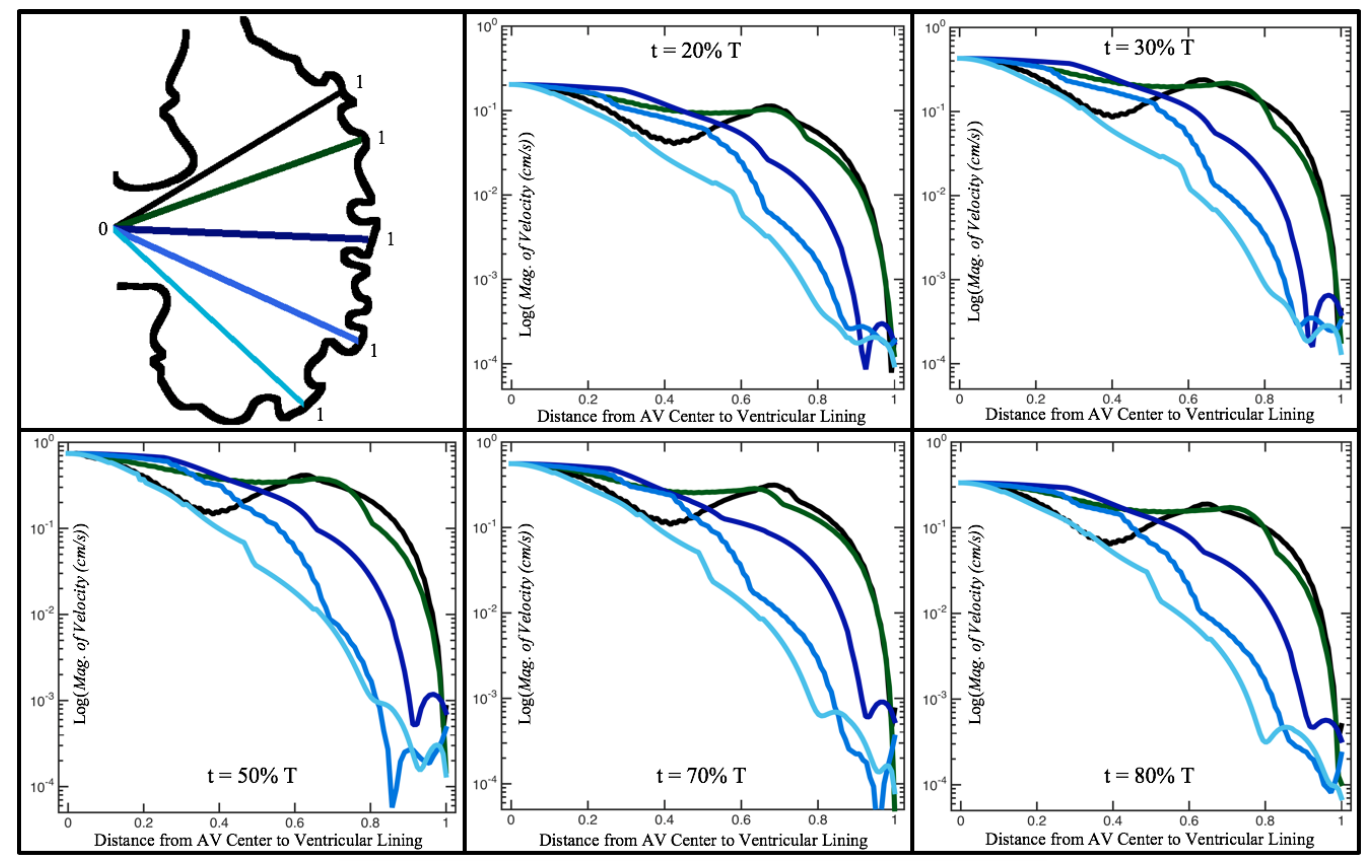

Figure 10. Plots showing how the log magnitude of velocity decays from the center of the AV canal to the ventricle wall for five different lines across the chamber using a $3 \mathrm{dpf}$ WT embryo's geometry at $R e=1$. The velocity decays until it reaches a trabeculae height away from the ventricle wall. Within pronounced trabecular valleys, the velocity increases before decaying further when measured closer to the ventricular lining. Each sub-figure corresponds to a different time during the pulsation cycle, described by a percentage of the pulsation period, $T$.

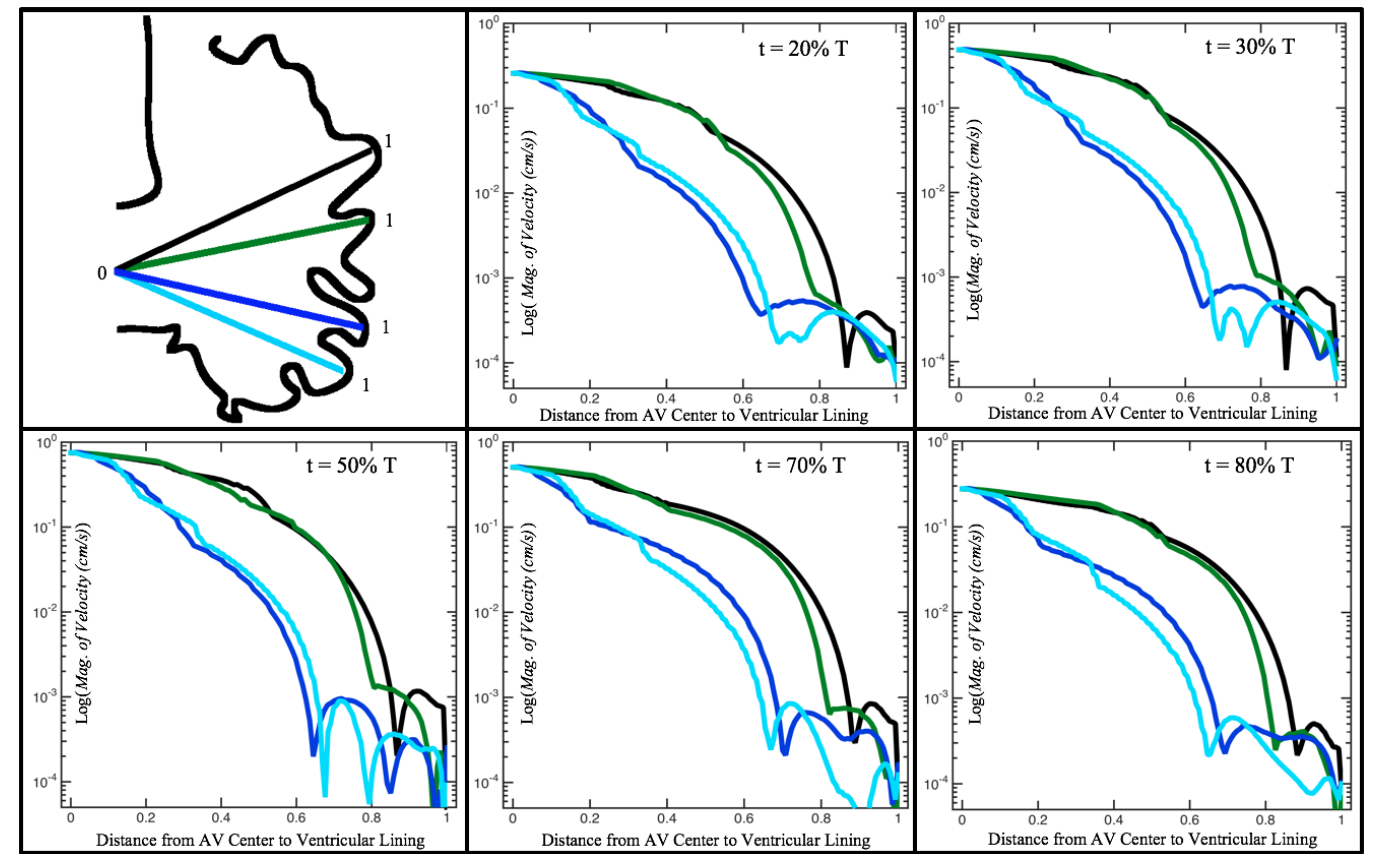

Figure 11. Plots showing how the log magnitude of velocity decays from the center of the AV canal to the ventricle wall for four different lines across the chamber using a $5 \mathrm{dpf}$ WT (I) embryo's geometry at $R e=1$. The velocity decays until it reaches a trabeculae height away from the ventricle wall. Within pronounced trabecular valleys, the velocity increases before decaying further when measured closer to the ventricular lining. Each sub-figure corresponds to a different time during the pulsation cycle, described by a percentage of the pulsation period, $T$. 


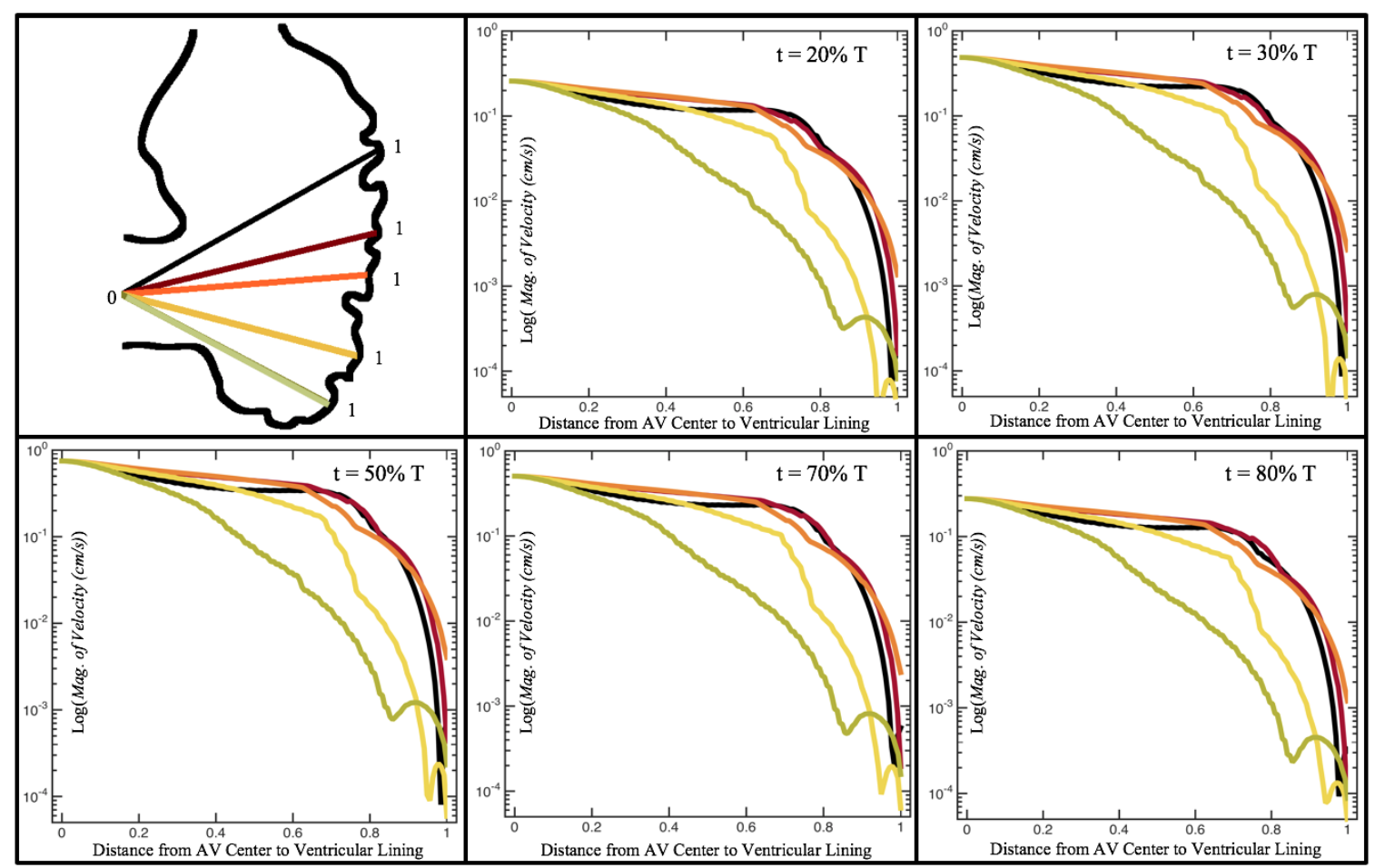

Figure 12. Plots showing how the log magnitude of velocity decays from the center of the AV canal to the ventricle wall for five different lines across the chamber using a $7 d p f$ ErbB2-inhibited embryo's geometry at $R e=1$. The velocity decays until it reaches a trabeculae height away from the ventricle wall. Within pronounced trabecular valleys, the velocity increases before decaying further when measured closer to the ventricular lining. Each sub-figure corresponds to a different time during the pulsation cycle, described by a percentage of the pulsation period, $T$.

Lastly for the case of pulsatile flow through realistic geometries, we computed the spatially-averaged wall shear stress (WSS) over the entire ventricular geometry, each pronounced trabeculae, and intertrabecular region, see Figure 13, as well as the oscillatory shear index (OSI). The OSI is a dimensionless metric of how aligned the WSS vector is with the time-averaged WSS (TAWSS) vector throughout one cardiac cycle. Recently, it has been suggested to be a possible regulatory mechanism of trabeculation itself [19]. Details regarding how WSS, TAWSS, and OSI were calculated can be found in Appendix D.

Figure 14 gives spatially-averaged magnitude of WSS over the trabeculae, intertrabecular regions, and entire ventricle for the $3 \mathrm{dpf}$ WT and $5 \mathrm{dpf}$ WT (I) zebrafish geometries. The intertrabecular regions experience slightly less $(\sim 10 \%)$ less spatially-averaged WSS than pronounced trabeculae. The majority of the WSS is felt elsewhere within the ventricle, e.g., near the AV-canal and outflow tract. See Appendix D.1 for a description of which trabeculae and intertrabecular regions were used for the analysis. 


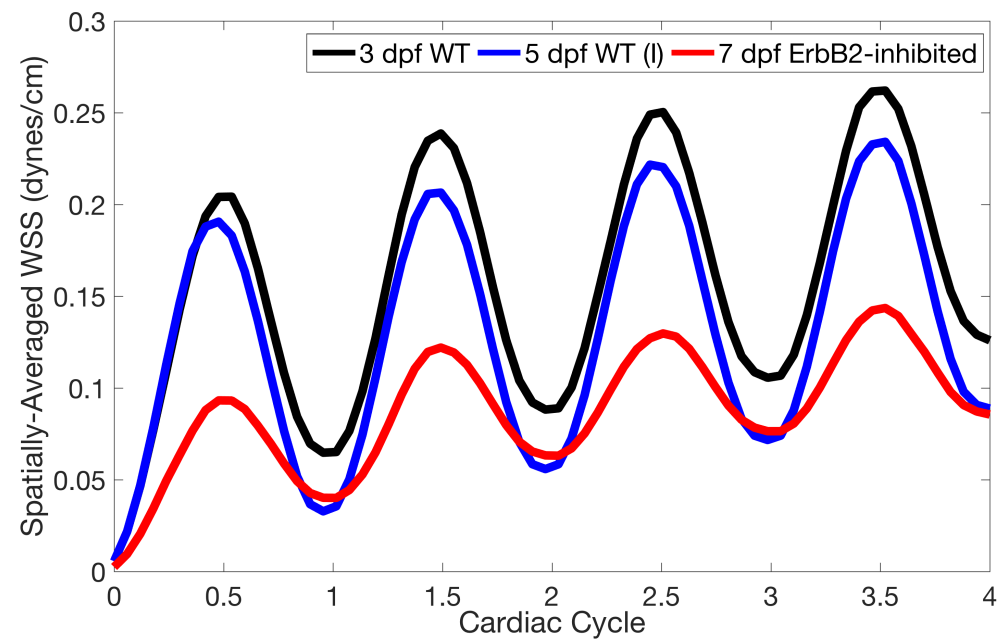

Figure 13. A plot showing the spatially-averaged magnitude of wall shear-stress (WSS) over the ventricle during the first 4 pulsation cardiac cycles for the $3 \mathrm{dpf}$ WT, $5 \mathrm{dpf}$ WT, and $7 \mathrm{dpf}$ ErbB2-inhibited zebrafish (ZF) for $R e=1$. The highest spatially-averaged WSS was in the $3 \mathrm{dpf}$ WT ZF with the least in the 7 dpf ErbB2-inhibited ZF geometry.

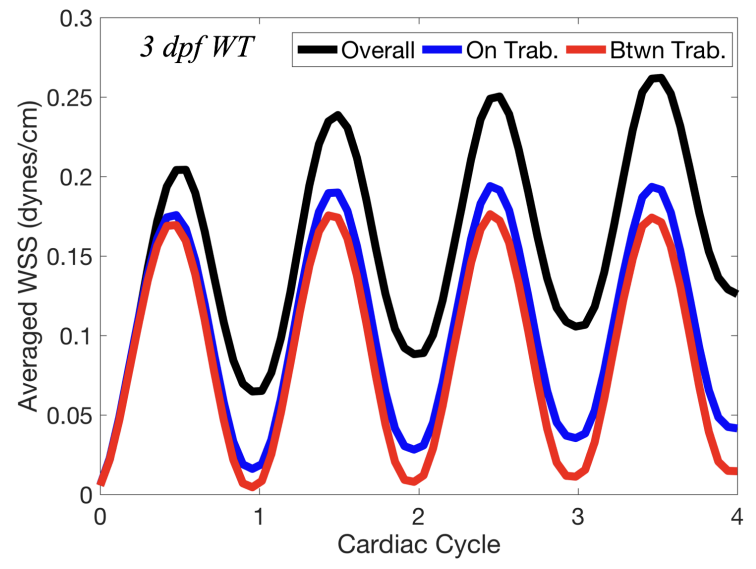

(a)

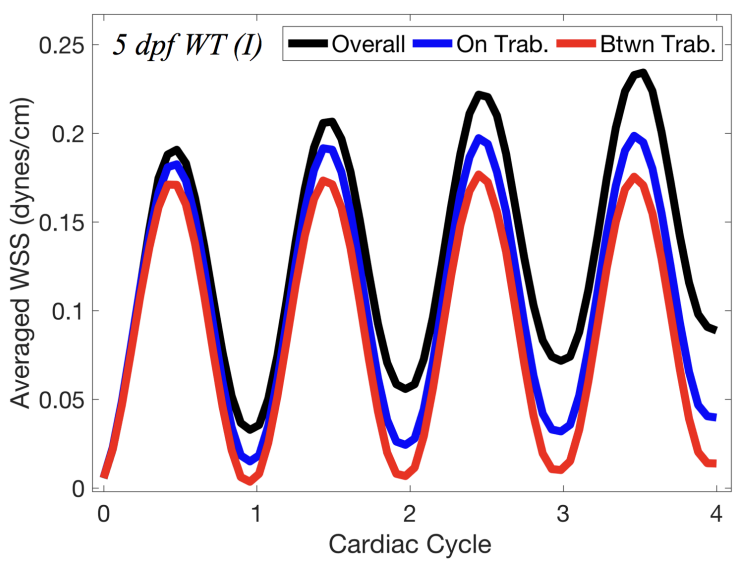

(b)

Figure 14. Plot giving the spatially-averaged magnitude of WSS over the trabeculae, the intertrabecular regions, and the entire ventricle during the first 4 pulsation cardiac cycles for the $3 \mathrm{dpf}$ WT (a) and $5 \mathrm{dpf}$ WT (I) (b) ZF for Re $=1$. In both cases, intertrabecular regions experience less WSS than the trabeculae themselves.

Figure 15 gives the OSI over the entire trabeculated ventricle for the 3 dpf WT, 5 dpf WT (I), and $7 \mathrm{dpf}$ ErbB2-inhibited zebrafish geometries. High OSI $\sim 0.4$ occur in both intertrabecular regions and on the trabeulae themselves. This is similar behavior to $[19,20]$ who reported high OSI values in intertrabecular regions as well; however, in our simulations, not all intertrabecular regions nor trabeculae have high OSI. In particular, those nearing the outflow tract (top of geometry) experience less OSI versus those opposite the lower end of the AV canal. 


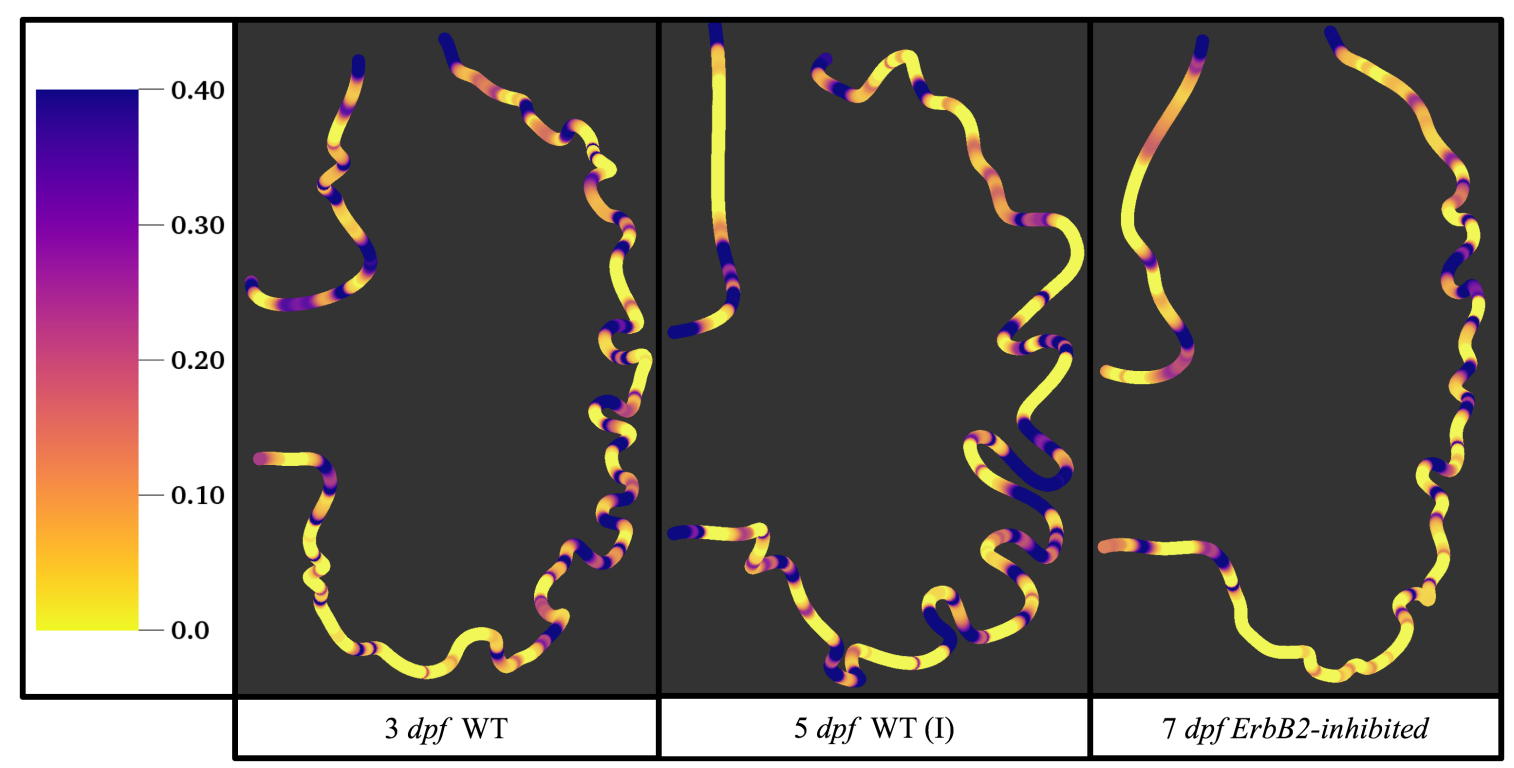

Figure 15. Plot giving the oscillatory shear index (OSI) over the ventricle during the 4th pulsation cardiac cycle for $R e=1$. High OSI occurs in intertrabecular regions and on the trabeculae themselves.

\subsection{Steady Flow through Idealized Trabeculated Chambers}

Figure 16 shows the flow field streamlines for the case of steady flow through an idealized trabeculated embryonic ventricle. The inflow condition is detailed in Appendix A. The numerical simulations span five orders of magnitude of $R e$, varying from 0.01 to 100 , while trabeculae heights were set to $0 \leq \frac{h_{T}}{b_{V}} \leq 0.16$. Note that the biologically relevant case is $\frac{h_{T}}{b_{V}}=0.08$

In the case of no trabeculae (left column), we find vortex formation only occurs for $R e \geq 15$, in agreement with the findings of [35]. Moreover, this result appears consistent with fluid dynamics literature on transitions to vortical flow via a channel with an expanded region [48,49]. Shen et al. [48] and Mizushima et al. [49] both use rectangular cavities; however, similar transitions to vortical flow occur at consistent $R e$. For $R e \leq 10$, the flow bends around the cavity and no flow separation occurs, that is, there are no transitions to vortical flow patterns. Hence, fluid mixing is not enhanced at for $R e \leq 10$ and flow reversal does not occur. As $R e$ is increased to 20, flow reversal occurs and a closed vortex is present along the left side of the cavity. The stagnation point is located between the orange and blue streamlines. To the left of this stagnation point, the flow moves along the endocardium from the right to left. To the right of the stagnation point, the flow moves right to left. As Re is further increased, the stagnation point moves to the right, and the intracardial vortex becomes larger until it becomes as large as the cavity itself for $R e \sim 100$.

When half-size biologically relevant trabeculae are introduced into the model $\left(\frac{h_{T}}{b_{V}}=0.04\right)$, similar flow fields emerge for the case of $R e \leq 10$. Although geometric perturbations now exist along the cavity lining, no flow separation occurs, whether intracardially or intertrabecularly, illustrating no vortical flow patterns nor enhanced fluid mixing at these trabeculae heights for $R e \leq 10$. For $R e=20$, we see a similar intracardial vortex to the case without trabeculae; however, this vortex weaves along regions with trabeculae. Furthermore, there is an emergence of an independent closed vortex along the right side between two trabeculae. For $R e \geq 50$, we find the presence of one large intracardial vortex wrapping around each trabeculae.

For biologically relevant trabeculae heights, there are closed intertrabecular vortices for $R e$ as low as 0.1 , while no intracardial vortices are present at these lower $R e$. Shen et al. [48] saw a similar phenomenon with the formation of two vortices in the corners of their rectangular cavity. This is consistent with the formation of vortices near the only bottom of the trabeculae. On the other hand, interestingly, not all intertrabecular regions have closed vortices. As $R e$ is further increased from 
$R e=5$ to $R e=10$, the intertrabecular vortices grow in size. As in previous cases, a larger intracardial vortex forms at $R e=20$. On the left hand side of the cavity, there is smooth flow from left to right around the trabeculae. On the right side of the cavity, independent closed vortices form between the trabeculae, and the flow is from right to left. For $R e \geq 50$, a large intracardial vortex forms and no intertrabecular vortices persist.

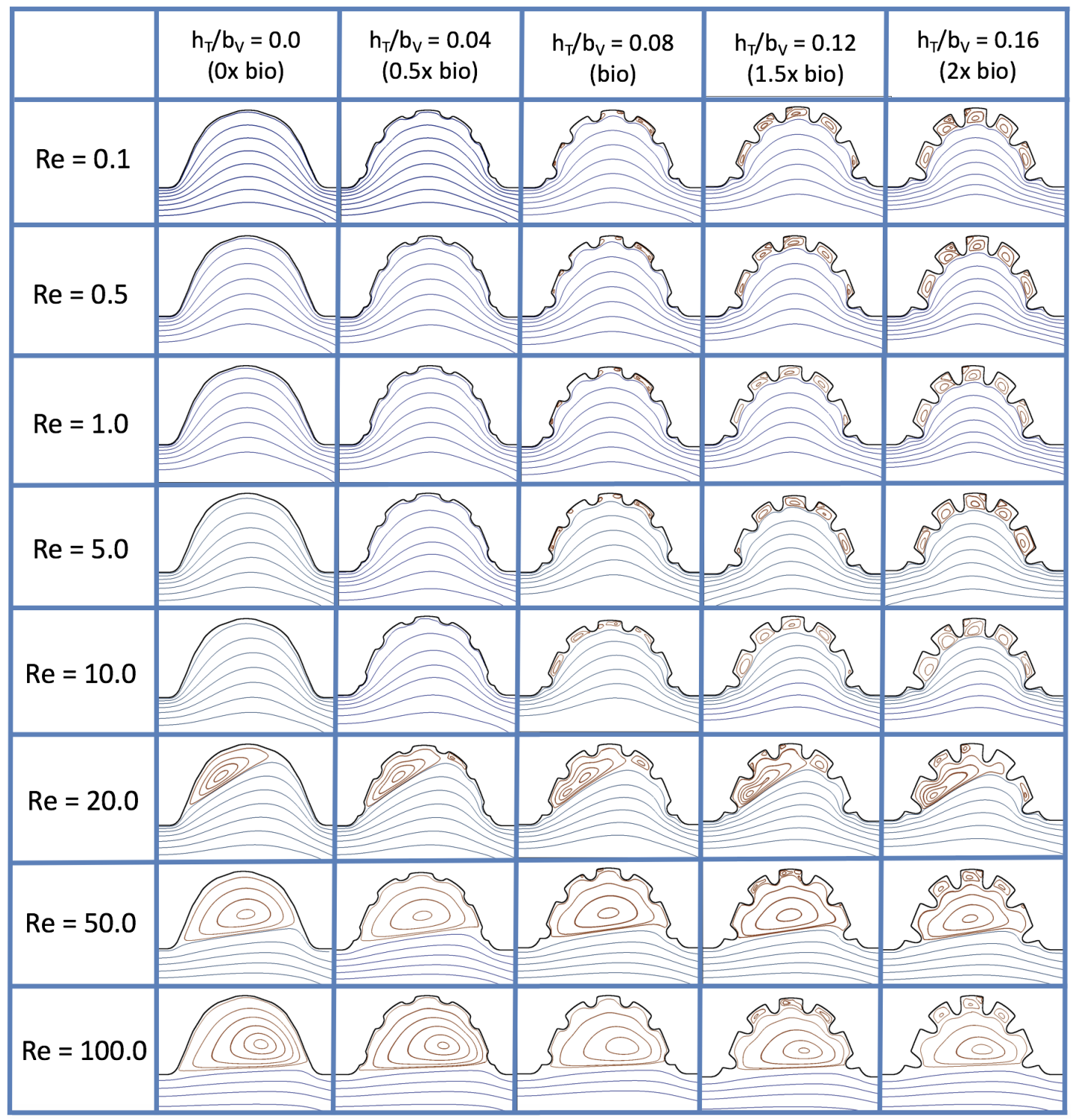

Figure 16. Streamline analysis performed for the case of steady flow into the trabeculated ventricle of a zebrafish at $4 \mathrm{dpf}$ for varying $R e$ and trabeculae heights.

For trabeculae heights higher than the biologically relevant range, there exist intertrabecular vortices for $R e$ as low as $R e=0.1$; however, compared to the previous biologically relevant case, there are vortices between every adjacent pair of trabeculae. Moreover, because the trabeculae extend further into the ventricular cavity, these vortices are larger than in previous cases. Intracardial vortices do not develop until $R e \geq 20$, where there is the presence of one large intracardial vortex on the left side of the cavity. When $R e=20$ and $\frac{h_{T}}{b_{V}}=0.12$, the intracardial vortex only wraps itself around the first four trabeculae with flow moving from left to right. A single intertrabecular vortex forms in the fourth trabecular valley. When $R e=20$ and $\frac{h_{T}}{b_{V}}=0.16$, the intracardial vortex extends over the left five trabeculae, with an intertrabecular vortex only in the last valley between trabeculae on the right 
side. For $R e \geq 50$, there is the formation of a large intracardial vortex extending throughout the cavity. However, both the trabeculae heights and $R e$ are large enough that this vortex does not wrap around each trabeculae, and intertrabecular vortices are able to form.

Furthermore, for the biologically relevant case of $R e=1$, Figure 17 illustrates the magnitude of velocity from the intracardial center to the ventricular lining for various intertrabecular regions and trabeculae heights. It is clear that, for larger trabeculae heights, the velocity decays moving away from the intracardial center at a faster rate than those cases with shorter or no trabeculae.

When the trabeculae are at biologically relevant heights or larger, the velocity decays from the middle of the ventricle until a distance of that height away from the ventriclular lining. Within that trabecular valley, the velocity actually increases before further decaying when measured closer to the ventricular lining. This shows that a local minima in velocity magnitude exists, suggesting that trabeculae morphology plays an important role in governing the fluid dynamics nearing the ventricular lining. This supports that there is intertrabecular vortex formation in these cases, as shown in Figure 16. The velocities measured in such intertrabecular regions are less than those shown in the cases with no or smaller trabeculae.

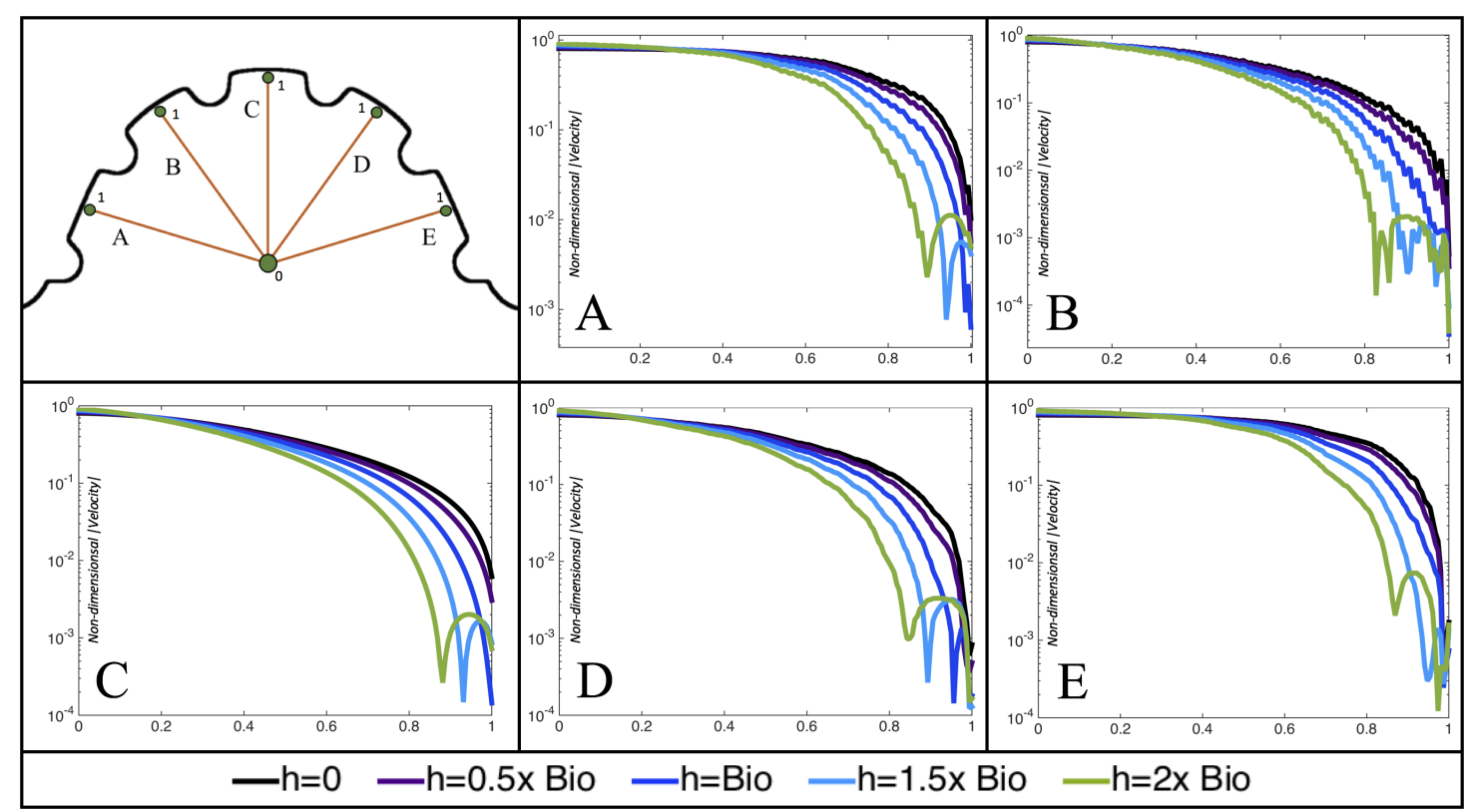

Figure 17. Measurements of the non-dimensional magnitude of velocity are provided for the $R e=1$ case quantified along a line from the intracardial center (labeled " 0 ") and extending to the ventricular lining (labeled "1") for various intertrabecular regions and trabeculae heights. The velocity magnitude strictly decreases from the center until around the neighboring trabeculae heights. The velocity magnitude then increases towards the center of the intratrabecular region, in some cases an order of magnitude, before dropping towards zero at the ventricle lining.

Interestingly, similar results are seen in the $R e=10$ case, see Figure A6 in Appendix E. However, in the case of $R e=100$, slightly different quantitative behavior is observed, see Figure 18. Note that the local minimum in velocity magnitude is still observed at the neighboring trabeculae height away from the ventricular wall, followed by an increase and then decrease as one moves closer to the wall. In contrast, as you measure away from the intracardial center, the velocity magnitude decreases (as before), but it also increases and then decreases before reaching the trabeculae height distance from the ventricle wall. This is due to the presence of a large intracardial vortex that forms, as illustrated in Figure 16. 


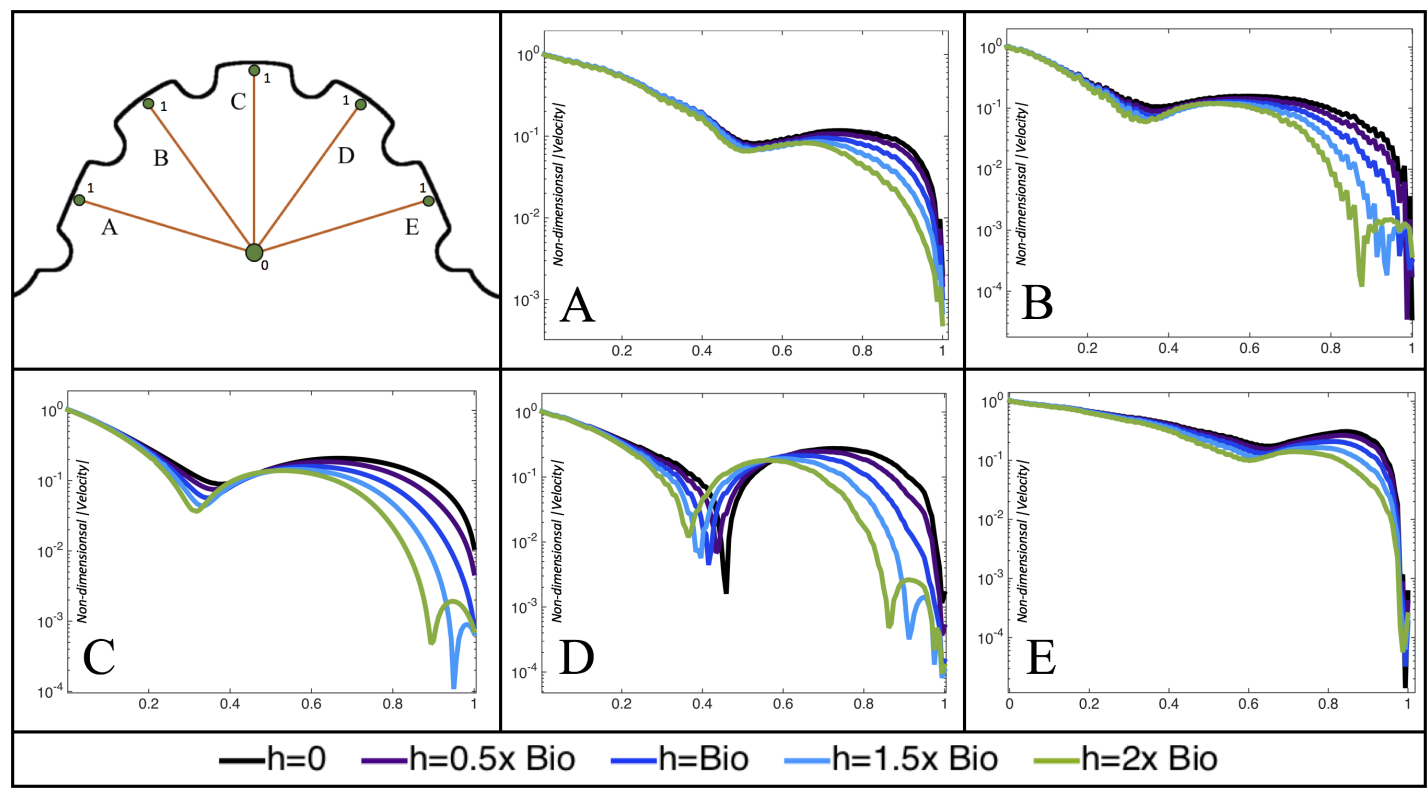

Figure 18. Measurements of the non-dimensional magnitude of velocity are provided for the $R e=100$ case quantified along a line from the intracardial center (labeled " 0 ") and extending to the ventricular lining (labeled "1") for various intertrabecular regions and trabeculae heights. Each subfigure (A-E), corresponds to a different line between the intracardial center and ventricular lining in which the magnitude of velocity is measured. The velocity magnitude decreases from the center and then increases before decreasing again as one approaches a distance from the wall that is equal to the neighboring trabeculae heights. As one moves between the trabeculae, the velocity magnitude again increases, in some cases an order of magnitude, before dropping towards zero at the ventricle lining.

\subsection{Pulsatile Flow through Idealized Trabeculated Chambers}

Next, we consider the same idealized trabeculated ventricle but use a pulsatile inflow condition, as described in Appendix A. The pulsation frequency is given by a dimensionless frequency close to that reported for a $4 \mathrm{dpf}$ embryonic zebrafish $(\tilde{f}=1.0)$. The $\operatorname{Re}$ was set to $0.1,1.0,10$ and 100 . The dimensionless trabecular heights, $\frac{h_{T}}{b_{V}}$ were varied from 0.0 to 0.16 . Recall that the biologically relevant $R e$ is about one, and the biologically relevant dimensionless trabecular height is about 0.08 . Snapshots of the streamlines showing the flow patterns are taken from the last pulse for each simulation using either 9 or 10 time points.

Figures 19 and 20 show streamline plots taken at nine snapshots in time for lower Re cases, $\operatorname{Re}=0.1,1.0$, respectively. The streamlines are shown for $5 \%, 10 \%, 20 \%, 40 \%, 50 \%, 80 \%, 90 \%$, $95 \%$, and $100 \%$ of the pulse. Finer increments in time are given towards the beginning and end of the pulse to illustrate the rapidly changing dynamics. The $R e=0.1$ and 1.0 cases show similar results. For the majority of the pulse, the flow moves smoothly from left to right within the ventricle. In between the trabeculae, vortices form during most of the pulse if the dimensionless trabecular height is at least 0.08 . The development of these vortices causes the flow near the endothelial cells to move from right to left between the trabeculae and from left to right on the top of the trabeculae. In most cases, transient vortices form as the flow is decelerated at the end of the pulse. For $R e=1.0$, intertrabecular vortices form for small trabeculae, $\frac{h_{T}}{b_{V}}=0.04$, as the flow decelerates. This is different than in the steady inflow counterpart case in Section 3.3, where vortex formation only happened for biologically relevant heights or greater. 


\begin{tabular}{|c|c|c|c|c|c|}
\hline $\begin{array}{c}\mathrm{h}_{\mathrm{T}} / \mathrm{b}_{\mathrm{V}}=0.0 \\
(0 \mathrm{x} \text { bio) }\end{array}$ & & & & & \\
\hline $\begin{array}{c}\mathrm{h}_{\mathrm{T}} / \mathrm{b}_{\mathrm{V}}=0.04 \\
(0.5 \mathrm{x} \text { bio })\end{array}$ & & & & & \\
\hline $\begin{array}{c}\mathrm{h}_{\mathrm{T}} / \mathrm{b}_{\mathrm{V}}=0.08 \\
\text { (bio) }\end{array}$ & & & & & \\
\hline $\begin{array}{c}\mathrm{h}_{\mathrm{T}} / \mathrm{b}_{\mathrm{V}}=0.12 \\
(1.5 \times \text { bio }\end{array}$ & & & & & \\
\hline $\mathrm{h}_{\mathrm{T}} / \mathrm{b}_{\mathrm{v}}=0.16$ & & & & & \\
\hline & $5 \% \mathrm{~T}$ & $10 \% \mathrm{~T}$ & $20 \% \mathrm{~T}$ & $40 \% \mathrm{~T}$ & $60 \% \mathrm{~T}$ \\
\hline
\end{tabular}

\begin{tabular}{|c|c|c|c|c|}
\hline \multicolumn{5}{|c|}{$\begin{array}{c}\mathrm{h}_{\mathrm{T}} / \mathrm{b}_{\mathrm{V}}=0.0 \\
\text { (0x bio) }\end{array}$} \\
\hline \multicolumn{5}{|l|}{$\begin{array}{c}\mathrm{h}_{\mathrm{T}} / \mathrm{b}_{\mathrm{V}}=0.04 \\
(0.5 \mathrm{x} \text { bio })\end{array}$} \\
\hline \multicolumn{5}{|l|}{$\begin{array}{c}h_{T} / b_{V}=0.08 \\
\text { (bio) }\end{array}$} \\
\hline \multicolumn{5}{|l|}{$\begin{array}{c}h_{T} / b_{V}=0.12 \\
(1.5 x \text { bio })\end{array}$} \\
\hline \multicolumn{5}{|l|}{$\begin{array}{c}\mathrm{h}_{\mathrm{T}} / \mathrm{b}_{\mathrm{V}}=0.16 \\
(2 \times \text { bio })\end{array}$} \\
\hline & $80 \% \mathrm{~T}$ & $90 \% \mathrm{~T}$ & $95 \% \mathrm{~T}$ & $100 \% \mathrm{~T}$ \\
\hline
\end{tabular}

Figure 19. Streamline analysis taken at different time points within a single pulsation cycle (described by a percentage of a single pulsation period, $T$ ) for the case of pulsatile flow into the trabeculated ventricle of a zebrafish at $4 \mathrm{dpf}$ for $R e=0.1$ and varying trabeculae heights.

Next, we considered the horizontal velocity extending from the intracardial center to the intertrabecular region directly above it in the case of $R e=1$. These results are shown in Figure 21. Flow velocities are at a minimum near the ventricular lining, with the magnitude of velocities decreasing at a slightly accelerated rate for larger trabeculae. On the other hand, at the intracardial center, the horizontal velocity increases with larger trabeculae in the middle of a pulsation cycle. As the pulsation cycle resides, it is clear that the flow direction also changes, giving rise to an intracardial vortex, as detailed in Figure 20. 


\begin{tabular}{|c|c|c|c|c|c|}
\hline $\begin{array}{c}\mathrm{h}_{\mathrm{T}} / \mathrm{b}_{\mathrm{V}}=0.0 \\
(0 \mathrm{x} \text { bio) }\end{array}$ & & & & & \\
\hline $\begin{array}{c}\mathrm{h}_{\mathrm{T}} / \mathrm{b}_{\mathrm{V}}=0.04 \\
(0.5 \times \text { bio })\end{array}$ & & & & & \\
\hline $\begin{array}{c}\mathrm{h}_{\mathrm{T}} / \mathrm{b}_{\mathrm{V}}=0.08 \\
\text { (bio) }\end{array}$ & & & & & \\
\hline $\begin{array}{c}\mathrm{h}_{\mathrm{T}} / \mathrm{b}_{\mathrm{V}}=0.12 \\
(1.5 \mathrm{x} \text { bio })\end{array}$ & & & & & \\
\hline $\mathrm{h}_{\mathrm{T}} / \mathrm{b}_{\mathrm{V}}=0.16$ & & & & & \\
\hline & $5 \% \mathrm{~T}$ & $10 \% \mathrm{~T}$ & $20 \% \mathrm{~T}$ & $40 \% \mathrm{~T}$ & $60 \% \mathrm{~T}$ \\
\hline
\end{tabular}

\begin{tabular}{|c|c|c|c|c|}
\hline $\begin{array}{c}\mathrm{h}_{\mathrm{T}} / \mathrm{b}_{\mathrm{V}}=0.0 \\
\text { (0x bio) }\end{array}$ & & & & \\
\hline $\begin{array}{c}\mathrm{h}_{\mathrm{T}} / \mathrm{b}_{\mathrm{V}}=0.04 \\
(0.5 \times \text { bio })\end{array}$ & & & & \\
\hline $\begin{array}{c}\mathrm{h}_{\mathrm{T}} / \mathrm{b}_{\mathrm{V}}=0.08 \\
\text { (bio) }\end{array}$ & & & & \\
\hline $\begin{array}{c}\mathrm{h}_{\mathrm{T}} / \mathrm{b}_{\mathrm{V}}=0.12 \\
(1.5 \mathrm{x} \text { bio })\end{array}$ & & & & \\
\hline $\begin{array}{c}\mathrm{h}_{\mathrm{T}} / \mathrm{b}_{\mathrm{V}}=0.16 \\
(2 \mathrm{x} \text { bio })\end{array}$ & & & & \\
\hline & $80 \% \mathrm{~T}$ & $90 \% \mathrm{~T}$ & $95 \% \mathrm{~T}$ & $100 \% \mathrm{~T}$ \\
\hline
\end{tabular}

Figure 20. Streamline analysis taken at different time points within a single pulsation cycle (described by a percentage of a single pulsation period, $T$ ) for the case of pulsatile flow into the trabeculated ventricle of a zebrafish at $4 \mathrm{dpf}$ for $R e=1.0$ and varying trabeculae heights.

Figure 22 shows the magnitude of velocity as as one moves along a line drawn from the top of the ventricle to the base of the middle intratrabecular region for five trabeculae heights five times during the pulse cycle. Note that, during a pulsation cycle, the snapshots taken during the middle of the pulse are similar to that of the steady inflow case for $R e=1$ shown in Figure 16. For larger trabeculae heights, the horizontal velocity reaches a minimum at distance that is about a trabecular height away from the ventricle wall. As one moves between the trabeculae, the magnitude of the velocity increases and then approaches as one nears the wall. These velocity profiles confirm the formation of vortices as shown in Figure 20. 


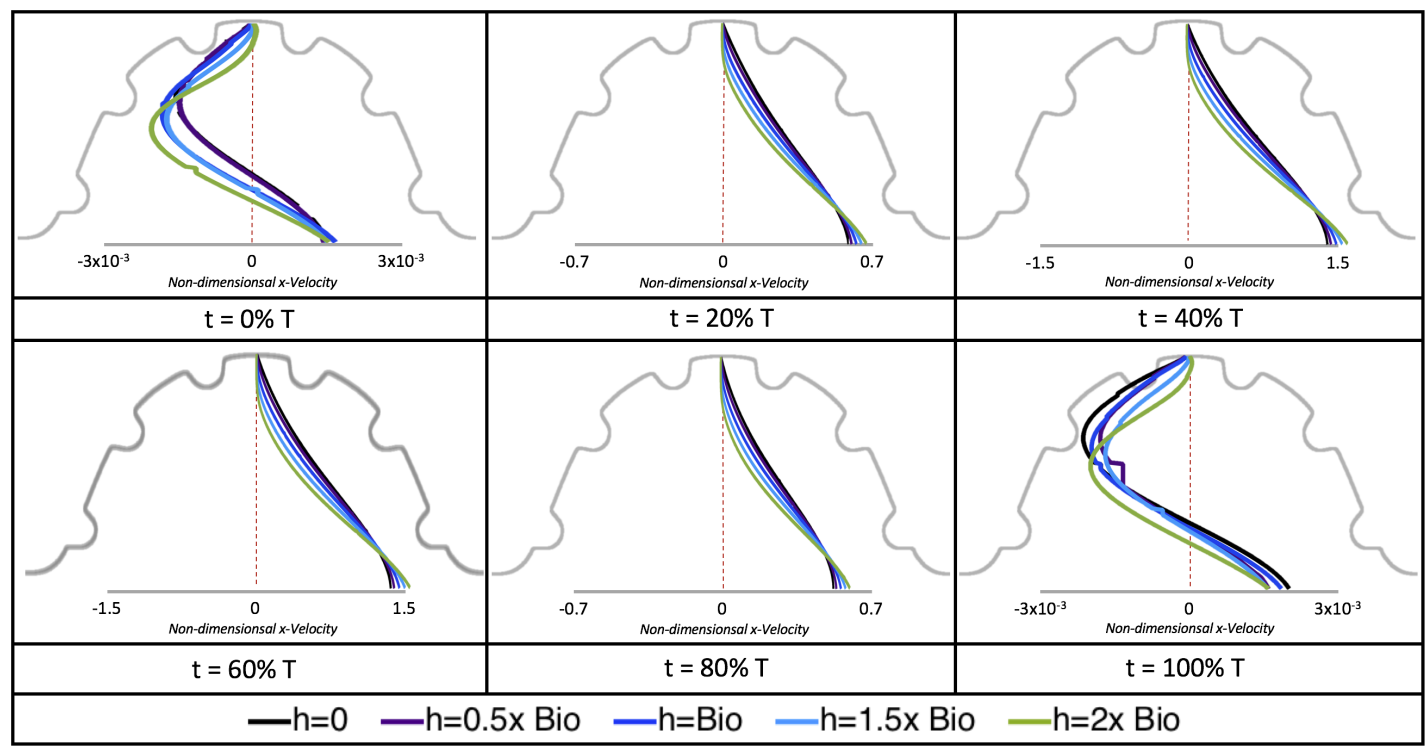

Figure 21. Snapshots during a pulsation cycle in the $R e=1$ case of the horizontal velocity measured from the intracardial center to the intertrabecular region directly above for multiple trabeculae heights. Each sub-figure corresponds to a different time point described as a percentage of the pulsation cycle, $T$.

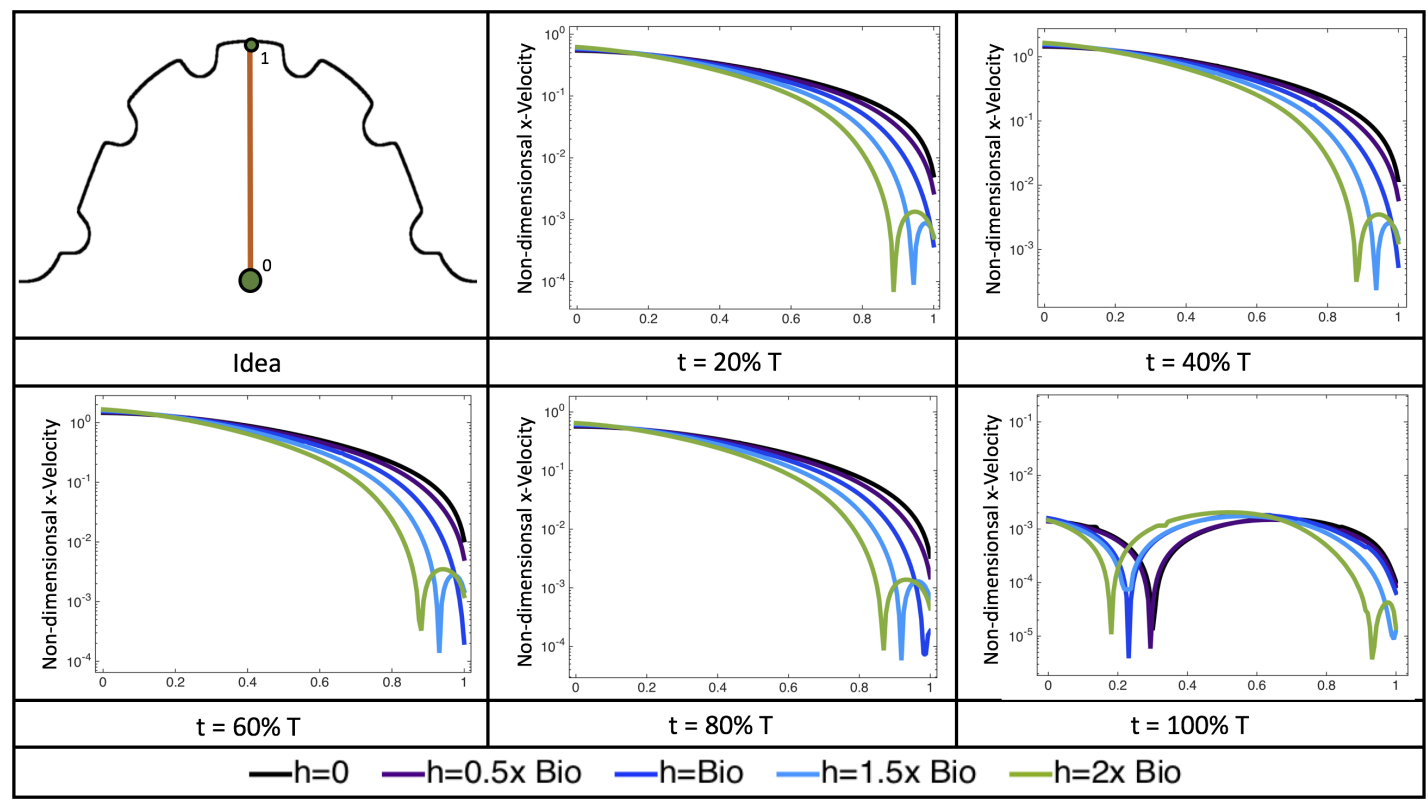

Figure 22. Snapshots during a pulsation cycle in the $R e=1$ case of the horizontal velocity measured from the intracardial center (labeled " 0 ") to the intertrabecular region (labeled " 1 ") directly above for multiple trabeculae heights. The trabeculae cause a drop in velocity as one nears the ventricular wall. Each sub-figure corresponds to a different time point described as percentge of the pulsation cycle, $T$.

Figure 23 shows streamline plots for $R e=10$ at ten evenly spaced times during a pulse. Intertrabecular vortices form during the first half of the pulse if the dimensionless trabecular height is at least 0.04 . For all geometries, intracardial vortices form during the last half of the pulse. The formation of the intracardial vortex annihilates the intertrabecular vortices, at least initially. The intracardial vortices form on the upstream side of the chamber, and grow to fill the entire chamber by the end of the pulse. The intertrabecular vortices form again towards the end of the pulse for $\frac{h_{T}}{b_{V}}=0.12,0.16$. Note that the presence of the intracardial vortex causes the intertrabecular vortices to change direction so that they spin clockwise (and the intracardial vortices spin counterclockwise). 


\begin{tabular}{|c|c|c|c|c|c|}
\hline $\begin{array}{c}\mathrm{h}_{\mathrm{T}} / \mathrm{b}_{\mathrm{V}}=0.0 \\
\text { (0x bio) }\end{array}$ & & & & & \\
\hline $\begin{array}{c}\mathrm{h}_{\mathrm{T}} / \mathrm{b}_{\mathrm{V}}=0.04 \\
(0.5 \mathrm{x} \text { bio })\end{array}$ & & & & & \\
\hline $\begin{array}{c}\mathrm{h}_{\mathrm{T}} / \mathrm{b}_{\mathrm{V}}=0.08 \\
\text { (bio) }\end{array}$ & & & & & \\
\hline $\begin{array}{c}\mathrm{h}_{\mathrm{T}} / \mathrm{b}_{\mathrm{V}}=0.12 \\
(1.5 \mathrm{x} \text { bio })\end{array}$ & & & & & \\
\hline $\begin{array}{c}\mathrm{h}_{\mathrm{T}} / \mathrm{b}_{\mathrm{V}}=0.16 \\
(2 \mathrm{x} \text { bio })\end{array}$ & & & & & \\
\hline & $10 \% \mathrm{~T}$ & $20 \% \mathrm{~T}$ & $30 \% \mathrm{~T}$ & $40 \% \mathrm{~T}$ & $50 \% \mathrm{~T}$ \\
\hline
\end{tabular}

\begin{tabular}{|c|c|c|c|c|c|}
\hline $\begin{array}{c}\mathrm{h}_{\mathrm{T}} / \mathrm{b}_{\mathrm{V}}=0.0 \\
(0 \mathrm{x} \text { bio })\end{array}$ & & & & & \\
\hline $\begin{array}{c}\mathrm{h}_{\mathrm{T}} / \mathrm{b}_{\mathrm{V}}=0.04 \\
(0.5 \mathrm{x} \text { bio })\end{array}$ & & & & & \\
\hline $\begin{array}{c}\mathrm{h}_{\mathrm{T}} / \mathrm{b}_{\mathrm{V}}=0.08 \\
\text { (bio) }\end{array}$ & & & & & \\
\hline $\begin{array}{c}\mathrm{h}_{\mathrm{T}} / \mathrm{b}_{\mathrm{V}}=0.12 \\
(1.5 \mathrm{x} \text { bio })\end{array}$ & & & & & \\
\hline $\begin{array}{c}\mathrm{h}_{\mathrm{T}} / \mathrm{b}_{\mathrm{V}}=0.16 \\
(2 \mathrm{x} \text { bio })\end{array}$ & & & & & \\
\hline & $60 \% \mathrm{~T}$ & $70 \% \mathrm{~T}$ & $80 \% \mathrm{~T}$ & $90 \% \mathrm{~T}$ & $100 \% \mathrm{~T}$ \\
\hline
\end{tabular}

Figure 23. Streamline analysis taken at different time points within a single pulsation cycle (described by a percentage of a single pulsation period, $T$ ) for the case of pulsatile flow into the idealized trabeculated ventricle of a zebrafish at $4 \mathrm{dpf}$ for $R e=10.0$ and varying trabeculae heights.

The spin direction is determined from the laminar flow, given in blue, moving left to right. When vortices first form in the intertrabecular regions, the vortices form moving counterclockwise. However, when a large intracardial vortex forms before intertrabecular vorticies, its direction is counterclockwise, which forces any intertrabecular vortices that form to move clockwise.

We also considered the horizontal velocity extending from the intracardial center to the intertrabecular region directly above for $R e=10$, as shown in Figure 24. Similarly quantitative behavior is seen as in the $R e=1$ case, see Figure 21; there is still significantly less flow in the intertrabecular region. However, the flow velocities are significantly different in the cavity below the intertrabecular region because of the presence of an intracardial vortex. It is clear that there is flow reversal given the differences in sign of the horizontal velocity. 


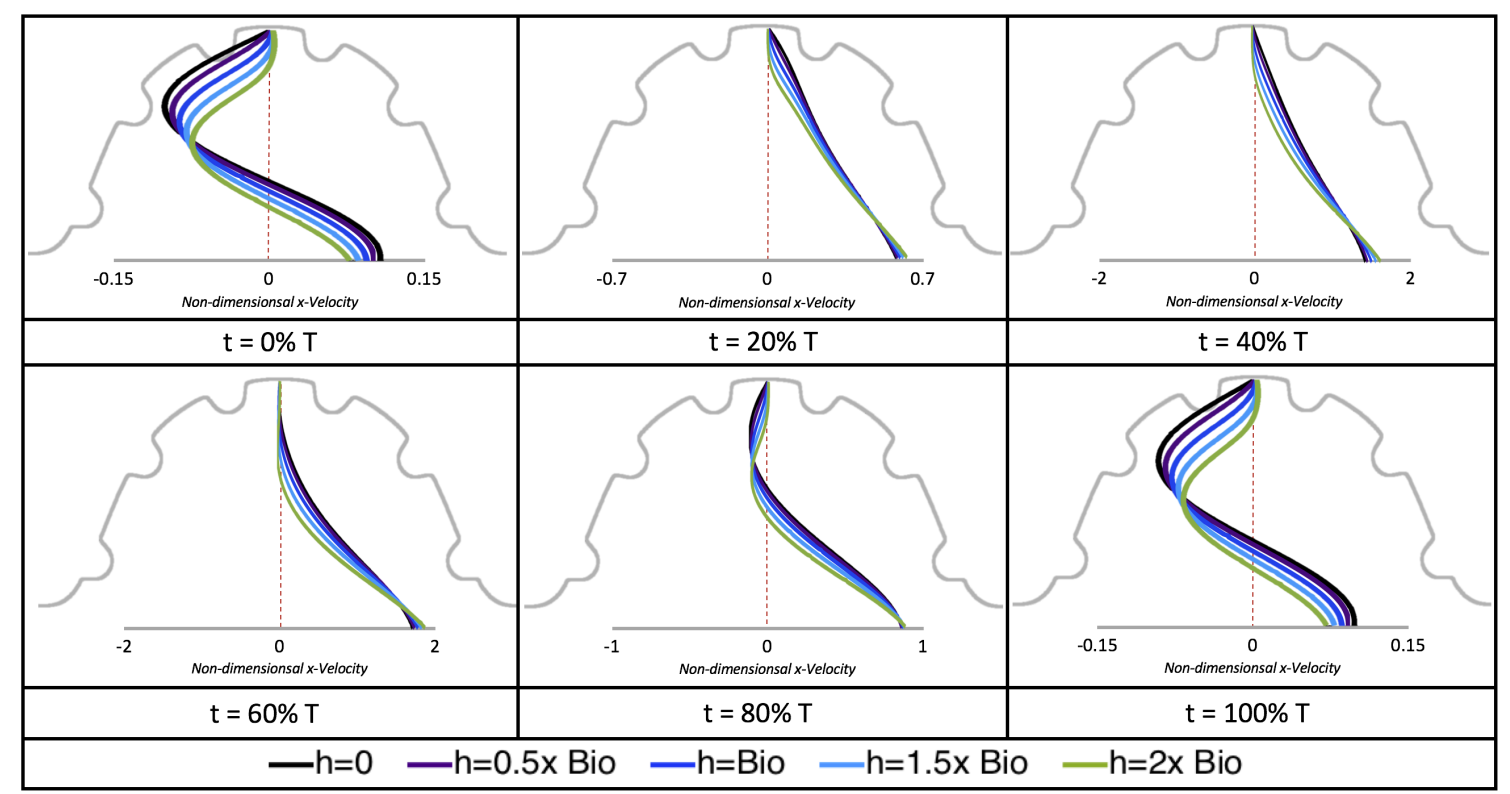

Figure 24. Snapshots during a pulsation cycle in the $R e=10$ case of the horizontal velocity measured from the intracardial center to the intertrabecular region directly above for multiple trabeculae heights. Each sub-figure corresponds to a different time point described as a percentage of the pulsation cycle, $T$.

The results of the inertial dominated case, $R e=100$, are shown in Figure 25. In all cases, a large intracardial vortex that fills the entire chamber is observed at the end of the pulse and the beginning of the next pulse. As the flow accelerates, the intracardial vortex is pushed downstream, and another intracardial vortex begins to form $(t=0.4 T-0.5 T)$. One or more oppositely spinning vortices form between the trabeculae or between the two counterclockwise spinning intracardial vortices when $t=0.5 T$. The upstream intracardial vortex combines with the original intracardial vortex such that one large intracardial vortex is observed around $t=0.7 \mathrm{~T}$. When this occurs, the oppositely spinning vortices are annihilated. For $\frac{h_{T}}{b_{V}} \geq 0.08$, oppositely spinning intertrabecular vortices reappear at the end of the pulse.

Due to the formation of large intracardial vortices, the horizontal velocity changes sign when measured from the center of the cavity and proceeds directly upward toward the ventricle lining (see Figure 26). Similar quantitative behavior is seen as in the $R e=10$ case. A substantial difference is the presence of a intracardial vortex that remains largely throughout the pulsation cycle. Moreover, similar to the other cases of $R e=1,10$, the velocity is significantly decreased within the intertrabecular region, even for $R e=100$. 


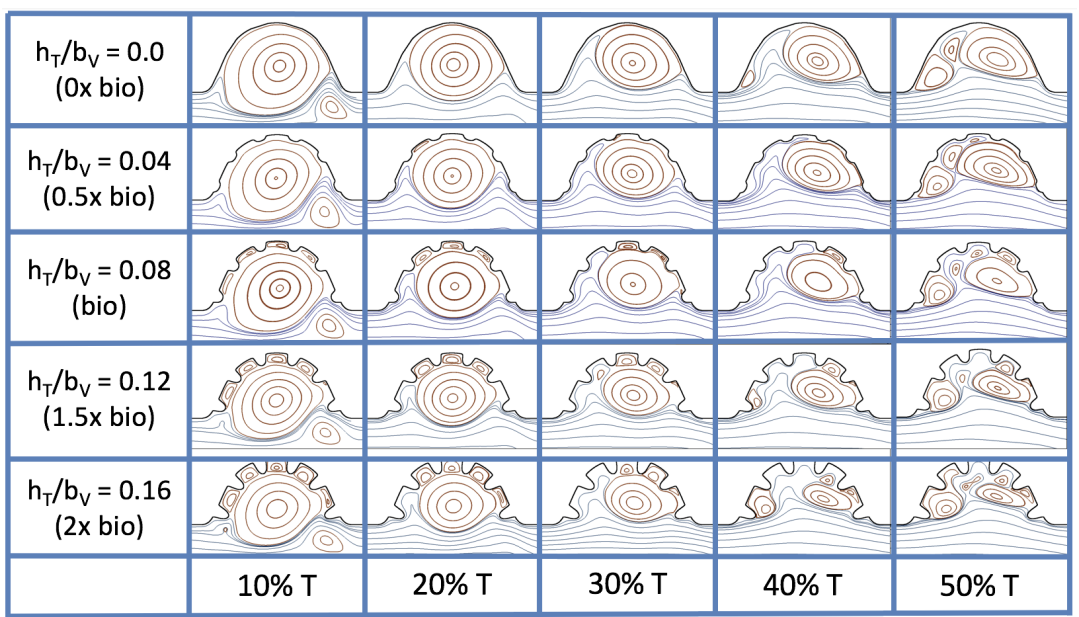

\begin{tabular}{|c|c|c|c|c|c|}
\hline \multicolumn{6}{|l|}{$\begin{array}{c}\mathrm{h}_{\mathrm{T}} / \mathrm{b}_{\mathrm{V}}=0.0 \\
(0 \mathrm{x} \text { bio) }\end{array}$} \\
\hline $\begin{array}{c}\mathrm{h}_{\mathrm{T}} / \mathrm{b}_{\mathrm{V}}=0.04 \\
(0.5 \mathrm{x} \text { bio })\end{array}$ & & & & & \\
\hline \multicolumn{6}{|l|}{$\begin{array}{c}h_{T} / b_{V}=0.08 \\
\text { (bio) }\end{array}$} \\
\hline \multicolumn{6}{|l|}{$\begin{array}{c}\mathrm{h}_{\mathrm{T}} / \mathrm{b}_{\mathrm{V}}=0.12 \\
(1.5 \mathrm{x} \text { bio) }\end{array}$} \\
\hline \multirow{2}{*}{$\begin{array}{c}\mathrm{h}_{\mathrm{T}} / \mathrm{b}_{\mathrm{V}}=0.16 \\
(2 \mathrm{x} \text { bio })\end{array}$} & & & & & \\
\hline & $60 \% \mathrm{~T}$ & $70 \% \mathrm{~T}$ & $80 \% \mathrm{~T}$ & $90 \% \mathrm{~T}$ & $100 \% \mathrm{~T}$ \\
\hline
\end{tabular}

Figure 25. Streamline analysis performed for the case of pulsatile flow into the trabeculated ventricle of a zebrafish at $4 \mathrm{dpf}$ for $R e=100$ and varying trabeculae heights.

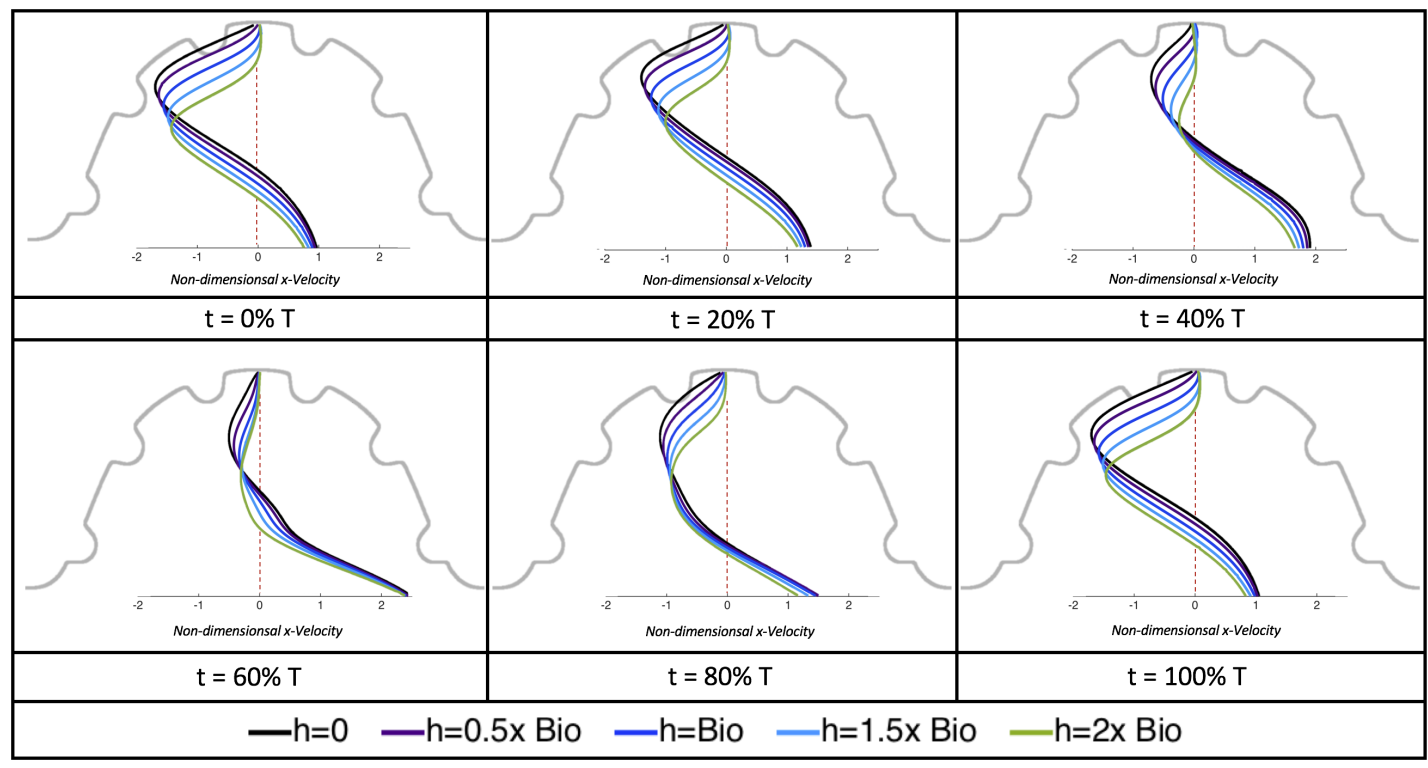

Figure 26. Snapshots during a pulsation cycle in the $R e=100$ case of the horizontal velocity measured from the intracardial center to the intertrabecular region directly above for multiple trabeculae heights. 


\section{Conclusions}

Two-dimensional immersed boundary simulations were used to solve for the flow fields within both biologically realistic geometries ( $3 \mathrm{dpf}, 80 \mathrm{hpf}$, two $5 \mathrm{dpf}$ WT zebrafish and a $7 \mathrm{dpf}$ ErbB2-inhibited zebrafish from [21]) and idealized models of trabeculated ventricles. Specifically, we investigated the intracardial and intertrabecular fluid dynamics searching for possible vortex formation and spatially-varying velocity gradients.

The primary result of our parametric study using simplified models of the trabeculated embryonic heart is that small changes in morphology and the effective viscosity of the blood can result in significant changes in bulk flow patterns as well as the magnitude and direction of shear felt by the endocardium. This presents an interesting challenge since each of these parameters is continuously changing during development. The results also highlight the importance of obtaining geometric descriptions of the developing heart with high spatial and temporal resolution. Small differences in morphology between individuals may also result in fundamentally different flow signals, underscoring the need to quantify variation. Furthermore, the effective viscosity of the blood is indirectly proportional to $R e$, and small changes in $R e$ may also result in drastic changes in flow direction and shear. The effective viscosity of the blood during development likely changes with hematocrit, which also changes from about $10-50 \%$ during development, and measurements of embryonic blood rheology are sparse [5].

This work focused specifically on the presence or absence of vortices given their significance to both the magnitude and direction of flow as well as the mixing patterns within the ventricle. When an intracardial vortex forms, the direction of the flow changes. When an intracardial vortex forms in unsteady flow, the direction of flow can change during the beat cycle, and the stagnation point moves along the cardiac wall. Since endothelial cells are known to sense and respond to changes in both magnitude and direction of flow, the formation and motion of these vortices could be important epigenetic signals.

The simulations revealed unexpected complexities in vortex dynamics as bulk flow moves through the chamber. In the cases of biologically realistic geometries, no large intracardial vortices developed at the biologically realistic fluid scale $(R e \sim 1)$ in the steady inflow case; in the pulsatile case, a large intracardial vortex formed when inflow velocities were minimal between pulses. However, for both steady and pulsatile inflow, intertrabecular vortices formed in pronounced trabeculated regions (Figures 5 and 7). While similar behavior was observed in both the steady and pulsatile inflow cases, more vortices formed in the unsteady flow cases in regions with less pronounced trabeculation. These results were consistent with the idealized trabeculation model as well (Figures 16 and 20). When vortices form in intertrabecular regions, the flow changes direction in those areas compared to the direction of bulk flow in the chamber. In cases where not all intertrabecular spaces have a vortex, the flow between different trabeculae will move in different directions.

In addition, as expected, the velocity tapers off when measuring flow speeds close to the ventricular lining. In most cases, the flow tapers off approximately three-orders of magnitude before reaching an intertrabecular region. Interestingly, in these regions where there is pronounced vortex formation, the velocity increases and then decreases as it nears the wall (for the pulsatile cases with realistic geometries, see Figures 10-12. Note that the corresponding steady inflow cases show similar trends). In the $5 \mathrm{dpf}$ WT (I) case, the velocity may increase an order of magnitude during portions of a pulsation cycle (see Figure 10), while, in other cases, the increase is modest, such as in the $3 \mathrm{dpf}$ WT case (see Figure 11). These results are quantitatively similar to those of the idealized model case at $R e=1$ for both pulsatile and steady inflow. The presence of trabeculae appears to control the fluid velocities in this regions, which helps govern the amount of shear-stress felt at the endothelial layer.

It is almost certainly the case that the exact flow patterns and magnitudes will not be exactly the same for realistic geometries with moving walls. Some similar features are observed between our simulations and previous work using idealized geometries with moving walls [40] as well as realistic geometries with moving walls $[19,20]$. In all cases, there is a severe reduction in flow magnitude in 
the intratrabecular regions and the presence of intratrabecular vortices exhibiting changes in flow direction and magnitude as a function of intratrabecular depth. The consequences of the reduction in flow magnitude and the presence of vortices are that lower shear stresses are felt at the endothelial wall than would be the case without trabeculae and that the direction of shear changes both spatially and temporally. Direct comparison of our results to work with a moving boundary and idealized geometry [40] shows that our simplified models do not capture the exact spatial and temporal pattern of flow reversals and shear, but they do demonstrate that these features exist and are sensitive to perturbations in kinematics and morphology.

The idealized model cases expanded the study by allowing us to easily manipulate the system to understand its sensitivity to vortex formation, due to its complex geometry, fluid scale, and inflow characteristics. In this vein, we increased the span of our larger fluid scale from $R e=0.1$ to $R e=100$, rather than simply the biological case of $R e=1$ for a $4 \mathrm{dpf}$ embryonic zebrafish heart. We also investigated equally sized idealized trabeculae in a chamber, and varied the heights from no trabeculae to trabeculae twice as large as biologically relevant according to Figure 3a, and included both pulsatile and steady inflow simulations to parse effects of unsteady flows on vortex formation.

Moreover, diversity of trabeculated hearts across the animal kingdom are far and wide, and may cover a large spectrum of length and fluid $(R e)$ scales. Even some invertebrate hearts contain ventricular trabeculation. In those invertebrates, their heart's morphology resembles that of lower vertebrates with sedentary lifestyles [50-52]. Some anthropods and mollusks contain ventiruclar trabeculae, such as blue crabs (Callinectes sapidus) [45], bar clams (Spisula solidissima) [53], oysters [54], snails [55], as well as octopus and squids [46]. However, any quantitative measurements detailing trabeculae morphology and flow measurements at varying time-points are unknown. It is possible that, in these hearts, the idealized simulations at higher $R e$ or for larger trabecular heights are relevant to these invertebrate hearts.

The idealized models showed that a large intracardial vortex forms around $R e \approx 20$ when steady flow is pushed through the chamber, while a similar sized vortex forms for $R e=10$ when the flow is pulsatile. In general, pulsatile flow lowers the $R e$ and trabeculae height needed to generate vortices. For both steady and unsteady flows as the trabeculae grow into the chamber, another bifurcation occurs in which small vortices form between each trabecula. Depending upon the Re and the morphology, the intertrabecular vortices can form without the presence of a large intracardial vortex (see Figure 16 for steady cases or Figures 19 or 20 for unsteady flow cases of $R e=0.1$ or 1.0 , respectively). In other cases, typically at higher $R e$, both the intracardial and intertrabecular vortices form, see Figures 23 or 25 , for unsteady inflow for $R e=10$ or 100 , respectively. In all corresponding cases, the presence of large intracardial vortices changes the direction of the intertrabecular vortices. Note that, in the biologically relevant case of $R e=1$ intracardial vortices do not form; this is consistent with the biologically accurate geometries as well. We do note that the exact patterns of intracardial and intratrabecular vortex formation may not hold for realistic geometries and pumping kinematics.

It is evident that there is a strongly coupled relationship between intracardial hemodynamics, genetic regulatory networks, and cardiac conduction. Besides contractions of the myocardial cells, which in turn drive blood flow, hemodynamics are directly involved in proper pacemaker and cardiac conduction tissue formation [56]. In addition, shear stresses are found to govern the conduction velocity distribution of action potentials within the myocardium [22]. Any changes in the emrbyonic heart's conduction properties will also affect the intracardial shear stresses, pressures, patterns of cyclic strains, and advection of morphogens. It is indeed a chicken and the egg scenario, especially when considering the first experiments that saw the importance of fluid dynamics in heart morphogenesis were performed in chicken embryos [57]. Dedicated initiatives to decipher exact cellular signalling pathways and genetic regulatory networks may be able to help further parse the causes of cardiac dysfunction. While CFD provides a robust framework to extract cardiac flow information (fluid velocities, shear stress distributions, pressures, etc.), coupling this data into a multi-scale cellular model is imperative to better understand the causes of many congenital heart diseases. 
Unfortunately, the exact mechanisms of mechanotransduction are not yet clearly understood [58,59]. Biochemical signals are thought to propagate throughout a pipeline of epigenetic signaling mechanisms, which may regulate of gene expression, cellular differentiation, proliferation, and migration [60]. In vitro studies have discovered that endothelial cells detect shear stresses as low as $0.2 \mathrm{dyn} / \mathrm{cm}^{2}$, resulting in up or down regulation of gene expressions [61]. Shear stresses around $\sim 8-15 \mathrm{dyn} / \mathrm{cm}^{2}$ are known to cause cytoskeletal rearrangement [62]. The aforementioned shear stresses reported in this paper and other CFD studies [20] are well in the range of those measured within embryonic hearts, $\sim 2 \mathrm{dyn} / \mathrm{cm}^{2}$ and $\sim 75 \mathrm{dyn} / \mathrm{cm}^{2}$ at approximately 1.5 and $4.5 \mathrm{dpf}$, respectively [3]. Mapping out the connection between fluid dynamics, the resulting ventricular stresses, electrophysiology, and the mechanical regulation of developmental regulatory networks are paramount to moving towards a more holistic understanding of heart development.

The simplified models used in this study permitted the exploration of a wide parameter space that includes the diversity of trabeculated hearts in vertebrate embryos and also in some invertebrates. There are obviously limitations in using such simplified models. Flow through the complex trabeculae of the ventricle is inherently three-dimensional. The flows generated by a beating ventricle will be different from pulsatile flows driven through a fixed geometry. Accordingly, our results should motivate the further development of more sophisticated three-dimensional models with complex geometry and moving boundaries. In the development of such models, our results illustrate how critical it is to obtain high resolution geometries and spatially and temporally resolved kinematics. Our results also point towards the consequences of variation in both geometry and blood rheology, which suggest that flow patterns may change drastically through development, between individuals, and across species.

Author Contributions: Conceptualization, N.A.B. and L.A.M.; Methodology and Software, N.A.B., D.R.D., and L.A.M.; Validation, N.A.B., D.R.D., A.N.L., and L.A.M..; Formal Analysis, Investigation, and Data Curation, N.A.B. and L.A.M; Writing-Original Draft Preparation, N.A.B., A.N.L., and L.A.M.; Writing-Review and Editing, N.A.B., A.N.L., L.A.S., J.L., and L.A.M.; Visualization, N.A.B.; Funding Acquisition, N.A.B. and L.A.M.

Funding: This project was funded by NSF DMS CAREER \#1151478 and NSF IOS \#1558052 awarded to L.A.M. Funding for N.A.B. and L.A.S. was provided from an National Institutes of Health T32 Grant (HL069768-14; PI, Nobuyo Maeda and Christopher Mack) and N.A.B. received funding from the NSF OAC-1828163 and the Support of Scholarly Activities Grant (TCNJ).

Acknowledgments: The authors would like to thank Steven Vogel for conversations on scaling in various hearts. We would also like to thank Lindsay Waldrop, Austin Baird, and William Kier for discussions on embryonic hearts.

Conflicts of Interest: The authors declare no conflict of interest.

\section{Abbreviations}

The following abbreviations are used in this manuscript:

$\begin{array}{ll}\text { DPF } & \text { Days Post Fertilization } \\ \text { HPF } & \text { Hours Post Fertilization } \\ \text { WT } & \text { Wild Type embryo } \\ \text { ZF } & \text { Zebrafish } \\ \text { AV } & \text { Atrioventricular } \\ \text { CFD } & \text { Computational Fluid Dynamics } \\ \text { Re } & \text { Reynolds Number } \\ \text { IB } & \text { Immersed Boundary Method } \\ \text { WSS } & \text { Wall Shear Stress } \\ \text { OSI } & \text { Oscillatory Shear Index }\end{array}$

\section{Appendix A. Immersed Boundary Method}

The immersed boundary method [63] was used to solve for the flow velocities within the geometric model from Section 2.2. The immersed boundary method (IB) has been successfully used to study the fluid dynamics of a variety of biological problems in the intermediate Reynolds number 
range, defined here as $0.01<R e<1000$ (see, for example, [64-69]). However, IB is capable of solving fully coupled fluid-structure interaction systems, here we only use it to solve fluid flow through complex model geometry. The model consists of stiff boundaries that are immersed within an incompressible fluid of dynamic viscosity, $\mu$, and density, $\rho$. The fluid motion is described using the full 2D Navier-Stokes equations in Eulerian form, given a

$$
\begin{gathered}
\rho\left(\frac{\partial \mathbf{u}(\mathbf{x}, t)}{\partial t}+\mathbf{u}(\mathbf{x}, t) \cdot \nabla \mathbf{u}(\mathbf{x}, t)\right)=-\nabla p(\mathbf{x}, t)+\mu \Delta \mathbf{u}(\mathbf{x}, t)+\mathbf{F}(\mathbf{x}, t), \\
\nabla \cdot \mathbf{u}(\mathbf{x}, t)=0
\end{gathered}
$$

where $\mathbf{u}(\mathbf{x}, t)=(u(\mathbf{x}, t), v(\mathbf{x}, t))$ is the fluid velocity, $p(\mathbf{x}, \mathbf{t})$ is the pressure, $\mathbf{F}(\mathbf{x}, t)$ is the force per unit volume (area in $2 D$ ) applied to the fluid by the immersed boundary, i.e., the ventricle geometry. The independent variables are the position, $\mathbf{x}=(x, y)$, and time, $t$. Equation (A1) is equivalent to the conservation of momentum for a fluid, while Equation (A2) is a condition mandating that the fluid is incompressible.

The interaction equations between the fluid and the immersed structure are given by

$$
\begin{gathered}
\mathbf{F}(\mathbf{x}, t)=\int \mathbf{f}(r, t) \delta(\mathbf{x}-\mathbf{X}(r, t)) d r \\
\mathbf{U}(\mathbf{X}(r, t), t)=\frac{\partial \mathbf{X}(r, t)}{\partial t}=\int \mathbf{u}(\mathbf{x}, t) \delta(\mathbf{x}-\mathbf{X}(r, t)) d \mathbf{x},
\end{gathered}
$$

where $\mathbf{X}(r, t)$ gives the Cartesian coordinates at time $t$ of the material point labeled by Lagrangian parameter $r, \mathbf{f}(r, t)$ is the force per unit area imposed onto the fluid by elastic deformations in the boundary, as a function of the Lagrangian position, $r$, and time, $t$. Equation (A3) applies a force from the immersed boundary to the fluid grid through a delta-kernel integral transformation. Equation (A4) sets the velocity of the boundary equal to the local fluid velocity.

The force equations are specific to the application. In a simple case where a preferred motion or position is enforced, boundary points are tethered to target points via springs. The equation describing the force applied to the fluid by the boundary in Lagrangian coordinates is given by $\mathbf{f}(r, t)$ and is explicitly written as

$$
\mathbf{f}(r, t)=k_{\text {target }}(\mathbf{Y}(r, t)-\mathbf{X}(r, t)),
$$

where $k_{\text {target }}$ is the stiffness coefficient, and $\mathbf{Y}(\mathbf{r}, \mathbf{t})$ is the prescribed Lagrangian position of the target structure. In all simulations, the immersed structure was held nearly rigid by applying a force proportional to the distance between the location of the actual boundary and the preferred position. The deviation between the actual and preferred positions can be controlled with the variable $k_{\text {target }}$.

The fluid flow is driven through the immersed boundary using either pulsatile parabolic inflows or a linear ramp to steady parabolic inflow at the location of the AV canal. The equations describing the specific inflow boundary conditions are given in Table A1. A partial Neumann outflow condition is enforced in the direction of flow at the outlet. This outflow condition is given as

$$
\left(\begin{array}{c}
\frac{\partial u}{\partial \hat{n}} \\
v
\end{array}\right)=\left(\begin{array}{l}
0 \\
0
\end{array}\right),
$$

where $u$ and $v$ are the $x$ - and $y$-components of the fluid velocity, respectively, and $\frac{\partial u}{\partial n}$ is the directional derivative of the $x$-component of the velocity taken in the direction normal to the boundary of the fluid domain. 
Table A1. Inflow boundary conditions for both simulations, one pertaining to parabolic steady inflow and the other corresponding to a parabolic pulsatile inflow. The parameters used for the boundary conditions are $f$, the non-dimensional frequency, which is matched to the zebrafish heart at 96 hpf, and $V_{i n}$, the maximum inflow velocity.

\begin{tabular}{cc}
\hline Case & Inflow BC \\
\hline Steady Inflow & $\mathbf{u}_{\text {in }}=\left(\begin{array}{c}\frac{V_{i n}}{d_{A V}^{2}} \tanh (2 t)\left(\frac{1}{4 d_{A V}^{2}}-y^{2}\right) \\
0\end{array}\right)$ \\
\hline Pulsatile Inflow & $\mathbf{u}_{\text {in }}=\left(\begin{array}{c}\frac{V_{i n}}{d_{A V}^{2}}|\sin (2 \pi f t)|\left(\frac{1}{4 d_{A V}^{2}}-y^{2}\right) \\
0\end{array}\right)$ \\
\hline
\end{tabular}

We used an adaptive and parallelized version of the immersed boundary method, IBAMR [70,71], to perform the simulations involving the highly idealized trabecular models, for both the steady inflow and pulsatile inflow cases. IBAMR is a $\mathrm{C}++$ framework that provides discretization and solver infrastructure for partial differential equations on block-structured locally refined Eulerian grids [72,73] and on Lagrangian (structural) meshes. IBAMR also includes infrastructure for coupling Eulerian and Lagrangian representations.

The Eulerian grid on which the Navier-Stokes equations were solved was locally refined near the immersed boundaries and regions of vorticity with a threshold of $|\omega|>0.05$. This Cartesian grid was organized as a hierarchy of four nested grid levels, and the finest grid was assigned a spatial step size of $d x=D / 1024$, where $D$ is the length of the domain. The ratio of the spatial step size on each grid relative to the next coarsest grid was 1:4. The temporal resolution was varied to ensure stability. Each Lagrangian point of the immersed structure was chosen to be $\frac{D}{2048}$ apart (twice the resolution of the finest fluid grid).

\section{Appendix B. Methods: Biologically Realistic Geometries}

We present two more computational geometries we will analyze for WT zebrafish embryos at $80 \mathrm{hpf}$ and $5 \mathrm{dpf}$ (II). Note that the $80 \mathrm{hpf}$ is close to the $3 \mathrm{dpf}$ time-point and the $5 \mathrm{dpf}$ (II) zebrafish illustrates natural variation in WT zebrafish from the embryo given in Figure 2.

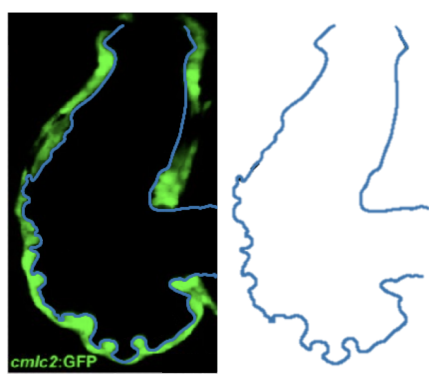

80 hpf wild-type

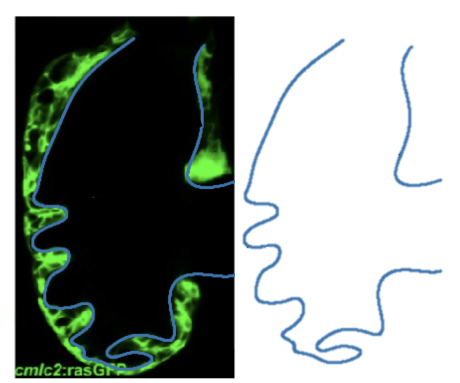

$5 d p f$ wild-type (II)

Figure A1. Extracting biologically realistic geometries of trabeculated geometries at different time-points from [21]. Geometries are given for a WT zebrafish at $80 \mathrm{hpf}$ and $5 \mathrm{dpf}$ (II).

\section{Appendix C. Results: Pulsatile Inflow for Biologically Realistic Geometries Results}

Here, we give more velocity measurements extending from the AV canal center to intertrabecular regions along the ventricular canal for cases of a 3 and $5 \mathrm{dpf}$ WT (I) and $7 \mathrm{dpf}$ ErbB2 inhibited zebrafish, Figures A2-A4, respectively. These figures are given to compare to their cases of steady inflow. Note that, in all cases, the velocity profiles taken at $50 \%$ of the pulsation cycle looks qualitatively identical to its corresponding steady inflow case. Note that this data was presented in Figures 10-12 as semi-log plots to illustrate the extent of the velocity decay as measured near the ventricular lining. 


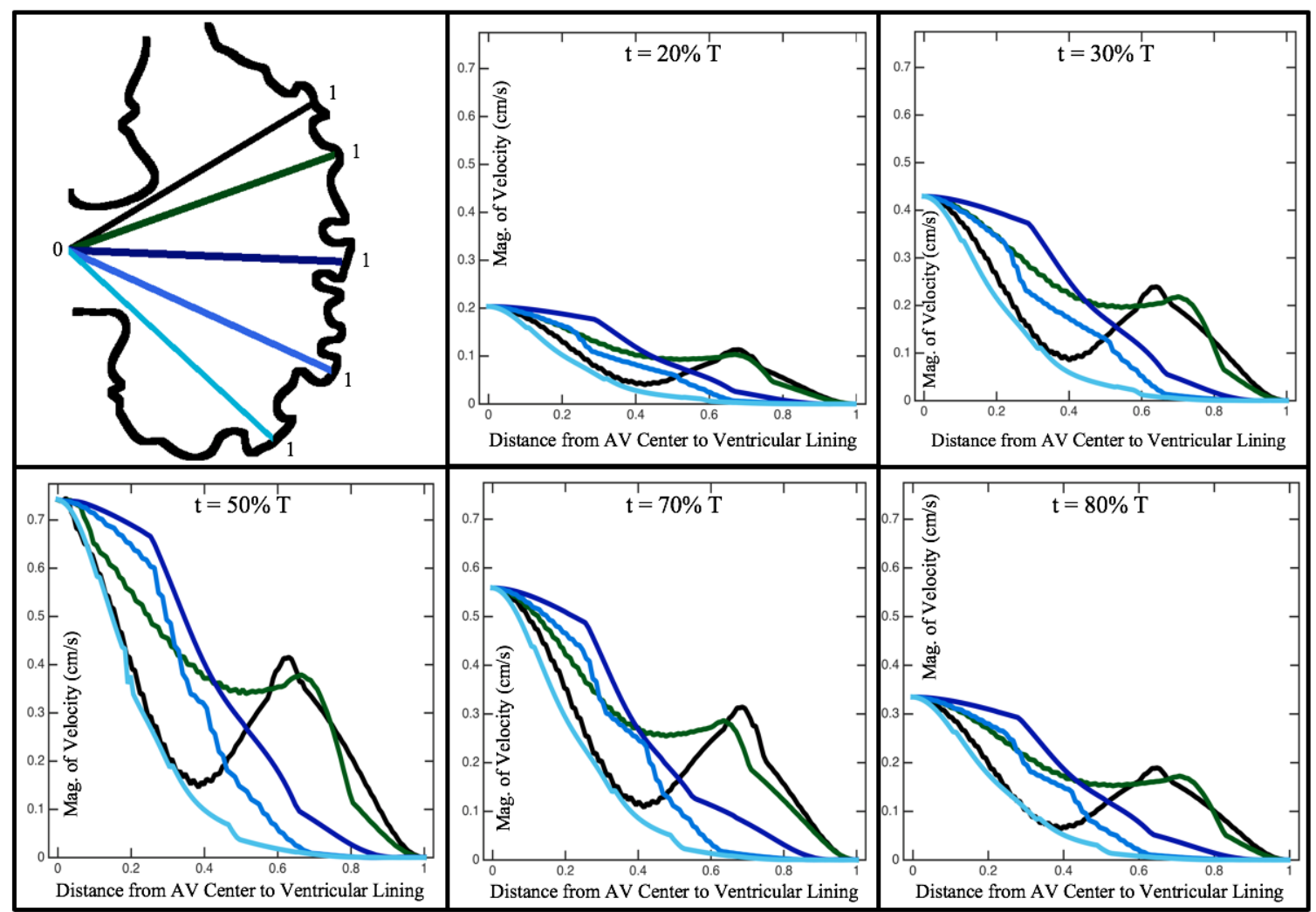

Figure A2. Plots showing how the magnitude of velocity decays from the center of the atrioventricular (AV) canal to the ventricle wall for four different lines across the chamber using a $3 \mathrm{dpf}$ WT embryo's geometry.

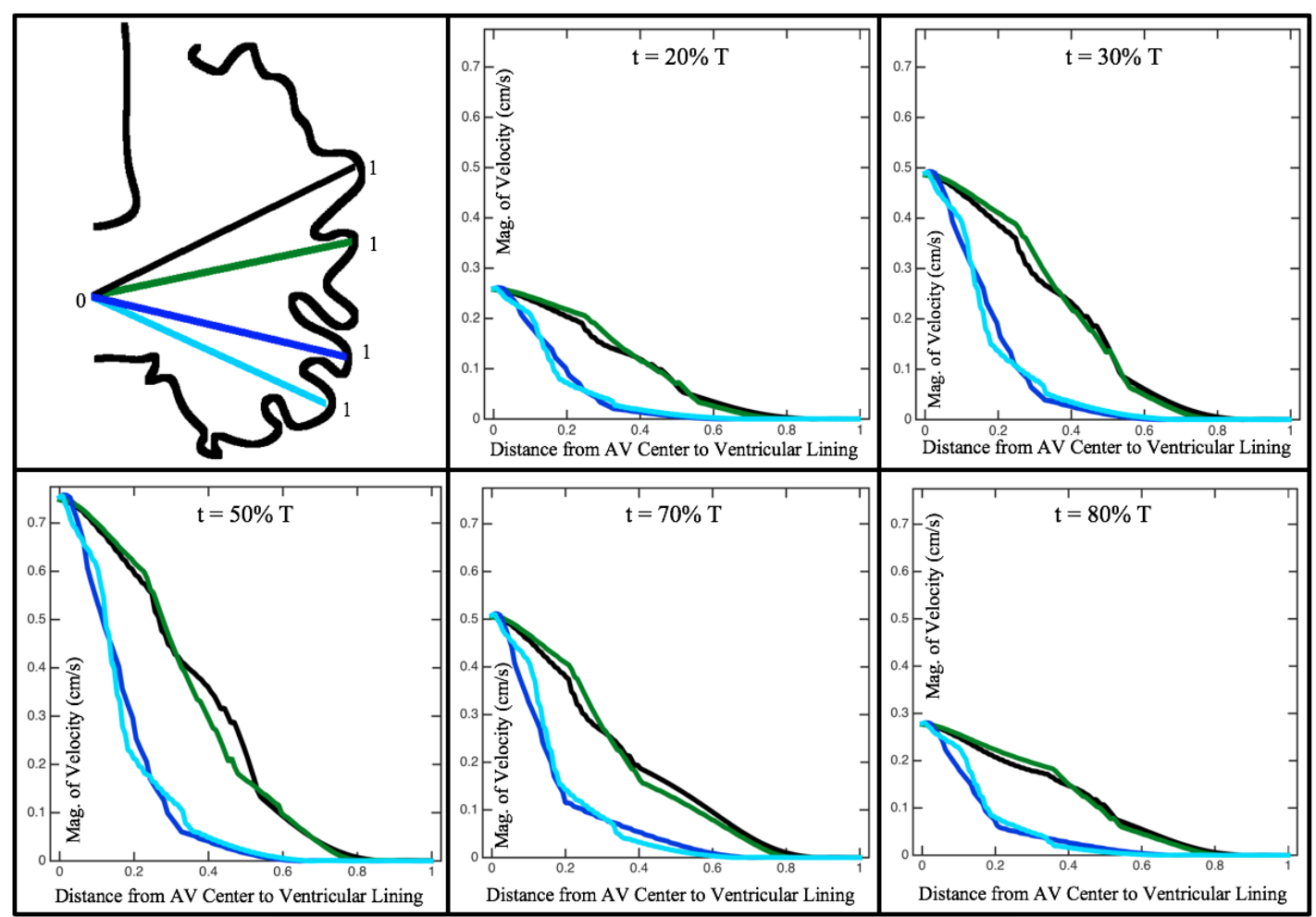

Figure A3. Plots showing how the magnitude of velocity decays from the center of the atrioventricular (AV) canal to the ventricle wall for four different lines across the chamber using a $5 \mathrm{dpf}$ WT (I) embryo's geometry. 


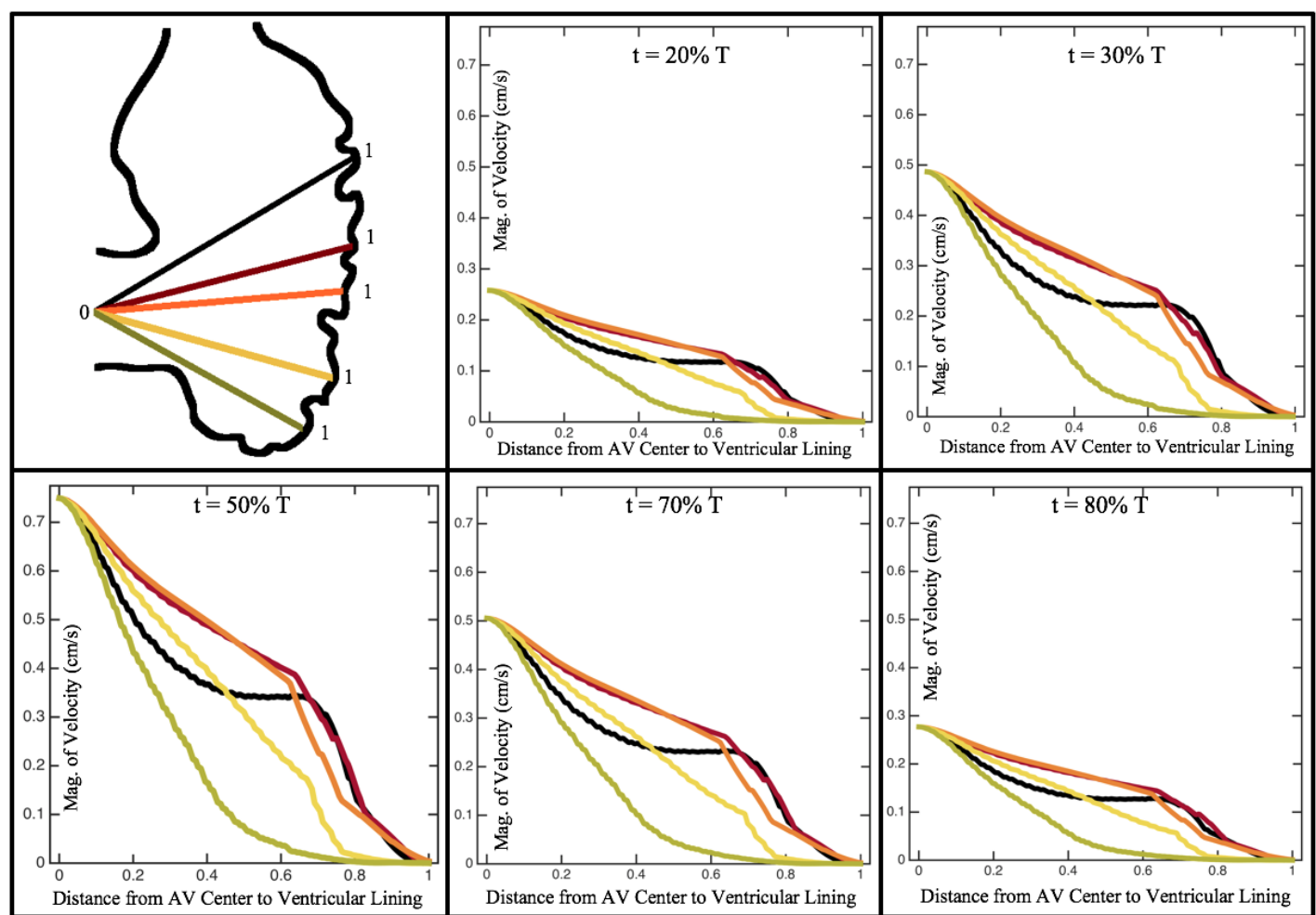

Figure A4. Plots showing how the magnitude of velocity decays from the center of the atrioventricular $(\mathrm{AV})$ canal to the ventricle wall for five different lines across the chamber using the ErbB2-inhibited emrbyo's geometry.

\section{Appendix D. Results: Computing WSS, TAWSS, and OSI}

WSS was computed as in [74], e.g., letting $\sigma=-p \mathbf{I}+\mu\left(\nabla \mathbf{u}+(\nabla \mathbf{u})^{T}\right)$ denote the fluid stress, the WSS, $\tau$ can be given by

$$
\boldsymbol{\tau}=\hat{t} \cdot[\boldsymbol{\sigma}] \cdot \hat{n}=-\frac{\hat{t} \cdot \mathbf{f}}{|\partial \mathbf{X} / \partial s|},
$$

where $\hat{t}$ and $\hat{n}$ is the anti-clockwise tangential and normal vector, respectively, $[\sigma]$ denotes the jump in the stress across the immersed boundary, and $|\partial \mathbf{X} / \partial s|$ gives the interfacial stretching.

TAWSS is calculated by temporally averaging the WSS, $\tau$, e.g.,

$$
\text { TAWSS }=\frac{1}{T} \int_{0}^{T}|\boldsymbol{\tau}| d t,
$$

where $T$ is the period of a cardiac cycle, as before.

OSI was computed as in [19]; e.g.,

$$
O S I=0.5\left(1-\frac{\left|\int_{0}^{T} \tau d t\right|}{\int_{0}^{T}|\boldsymbol{\tau}| d t}\right) .
$$

We accounted for noise in the force densities along the immersed boundary in two ways. First, the WSS and TAWSS were spatially averaged either across an entire trabeculae or intertrabecular region and the entire ventricle; we do not present shear-stress measurements at individual Lagrangian points but only at subsets of the Lagrangian domain. Second, the resulting OSI was spatially-averaged on each Lagrangian node using the closest five neighboring Lagrangian nodes. 
Appendix D.1. Results: Spatially-Averaged WSS for $3 \operatorname{dpf} W T$ and $5 \operatorname{dpf} W T$ (I)

Here, we detail how we computed the spatially-averaged WSS over the trabeculae, intertrabecular regions, and entire ventricle. This can be seen in Figure A5. Blue lines and red lines indicate regions used to compute trabecular and intertrabecular spatial-averages, respectively. Note that the entire ventricular geometry, including the trabecular in blue and intertrabecular regions in red, was used to compute the spatially-averaged WSS on the entire ventricle.

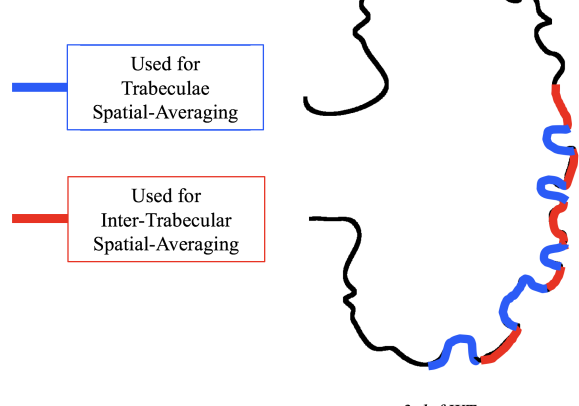

$3 d p f W T$

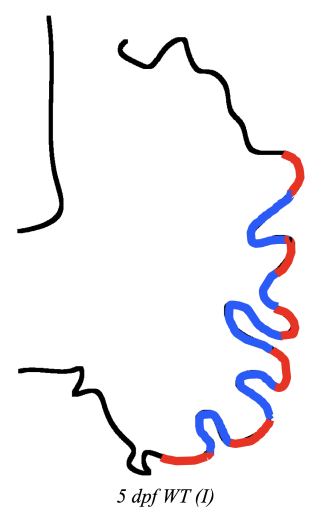

Figure A5. Plots detailing what regions were considered for spatially-averaging the WSS on the trabeculae and intertrabecular regions.

\section{Appendix E. Results: Steady Inflow for Idealized Geometry}

In this appendix, we present magnitude of velocity results in the idealized geometry case for $R e=10$ (see Figure A6 below). Figure A6 shows similar qualitative trends to that of the $R e=1$ case in Figure 17 of Section 3.3. However, differences arise in the actual quantitative magnitude of velocity measurements, where, in the $R e=10$ case, the velocity magnitudes are generally slightly greater.

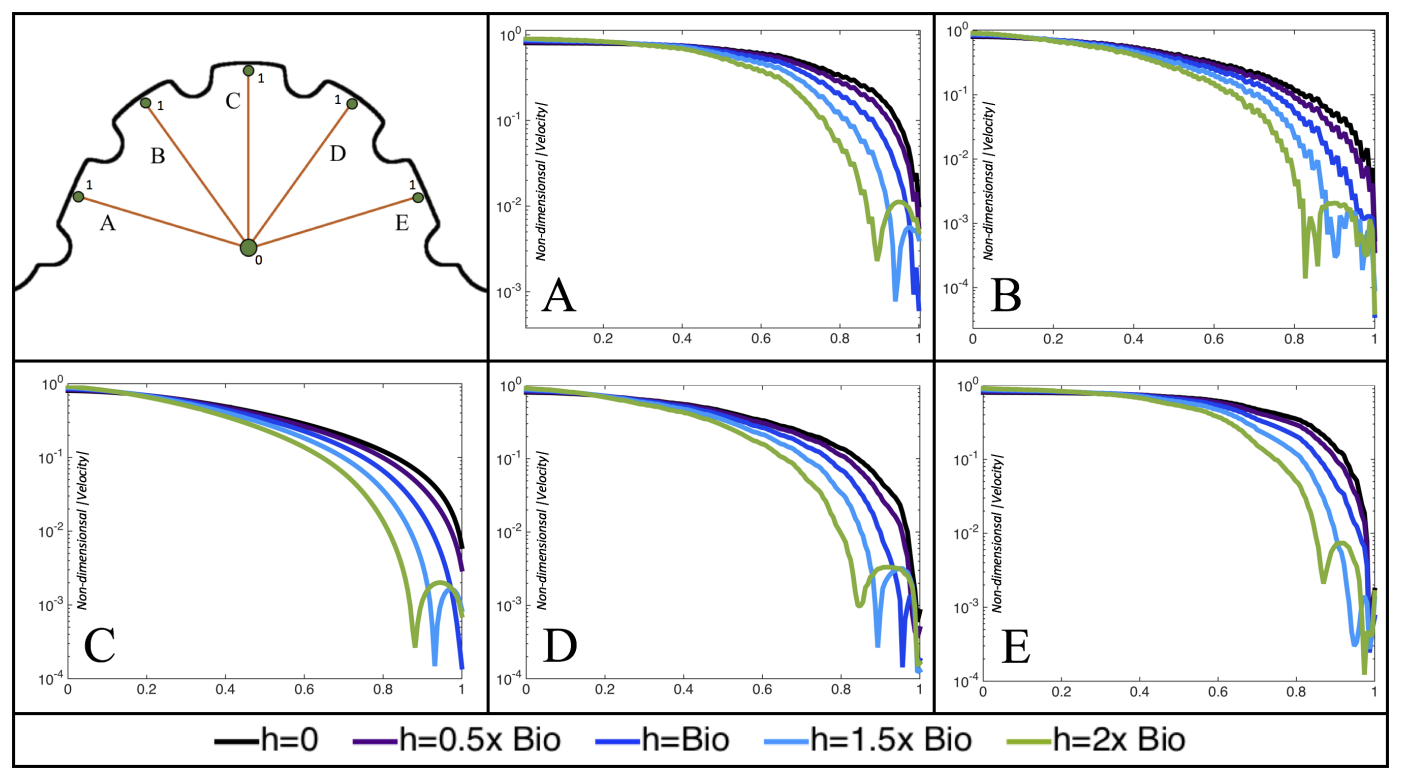

Figure A6. Measurements of the non-dimensional magnitude of velocity are provided for the $R e=10$ case quantified along a line from the intracardial center (labeled " 0 ") and extending to the ventricular lining (labeled "1") for various intertrabecular regions and trabeculae heights. The velocity strictly decreases from the center until around the neighboring trabeculae heights, in which the velocity increases, in some cases, an order of magnitude, before dropping towards zero at the ventricle lining. 


\section{References}

1. Burggren, W. What is the purpose of the embryonic heart beat? Or how facts can ultimately prevail over physiological dogma. Physiol. Biochem. Zool. 2004, 77, 333-345. [CrossRef] [PubMed]

2. Patterson, C. Even flow: Shear cues vascular development. Arterioscler. Thromb. Vasc. Biol. 2005, 25, 1761-1762. [CrossRef]

3. Hove, J.R.; Koster, R.W.; Forouhar, A.S.; Acevedo-Bolton, G.; Fraser, S.E.; Gharib, M. Intracardiac fluid forces are an essential epigenetic factor for embryonic cardiogenesis. Nature 2003, 421, 172-177. [CrossRef] [PubMed]

4. Lindsey, S.E.; Butcher, J.T.; Yalcin, H.C. Mechanical regulation of cardiac development. Front. Physiol. 2014, 5, 318. [CrossRef] [PubMed]

5. Santhanakrishnan, A.; Miller, L.A. Fluid Dynamics of Heart Development. Cell Biochem. Biophys. 2011, 61, 1-22. [CrossRef]

6. Icardo, J.M. Developmental biology of the vertebrate heart. J. Exp. Zool. 1996, 275, 144-161. [CrossRef]

7. Tarbell, J.M.; Weinbaum, S.; Kamm, R.D. Cellular fluid mechanics and mechanotransduction. Ann. Biomed. Eng. 2005, 33, 1719-1723. [CrossRef] [PubMed]

8. Taylor, A.D.; Neelamegham, S.; Hellums, J.D.; Smith, C.W.; Simon, S.I. Molecular dynamics of the transition from L-selectin- to beta 2-integrin-dependen neutrophil adhesion under defined hydrodynamic shear. Biophys. J. 1996, 71, 3488-3500. [CrossRef]

9. Cartwright, J.H.; Piro, O.; Tuval, I. Fluid dynamics in developmental biology: Moving fluids that shape ontogeny. HFSP J. 2009, 3, 77-93. [CrossRef]

10. Freund, J.B.; Goetz, J.G.; Hill, K.L.; Vermot, J. Fluid flows and forces in development: Functions, features and biophysical principles. Development 2012, 139, 1229-1245. [CrossRef]

11. Gruber, J.; Epstein, J. Development gone awry-congenital heart disease. Circ. Res. 2004, 94, $273-283$. [CrossRef]

12. Stekelenburg-de Vos, S.; Ursem, N.; Hop, W.; Wladimirioff, J.; Gittenberger-de Groot, A.C.; Poelmann, R.E. Acutely altered hemodynamics following venous obstruction in the early chick embryo. J. Exp. Biol. 2003, 206, 1051-1057. [CrossRef] [PubMed]

13. Kowalski, W.J.; Pekkan, K.; Tinney, J.P.; Keller, B.B. Investigating developmental cardiovascular biomechanics and the origins of congenital heart defects. Front. Physiol. 2014, 5, 408. [CrossRef] [PubMed]

14. Midgett, M.; Rugonyi, S. Congenital heart malformations induced by hemodynamic altering surgical interventions. Front. Physiol. 2014, 5, 287. [CrossRef] [PubMed]

15. Icardo, J.; Fernandez-Teran, A. Morphologic Study of Ventricular Trabeculation in the Embryonic Chick Heart. Acta Anat. 1987, 130, 264-274. [CrossRef] [PubMed]

16. Samsa, L.A.; Givens, C.; Tzima, E.; Didier, Y.; Stainer, R.; Qian, L.; Liu, J. Cardiac contraction activates endocardial Notch signaling to modulate chamber maturation in zebrafish. Development 2015, 142, 4080-4091. [CrossRef] [PubMed]

17. Granados-Riveron, J.; Brook, D. The Impact of Mechanical Forces in Heart Morphogenesis. Circ. Cardiovasc. Genet. 2012, 5, 132-142. [CrossRef]

18. Malone, M.; Sciaky, N.; Stalheim, L.; Klaus, H.; Linney, E.; Johnson, G. Laser-scanning velocimetry: A confocal microscopy method for quantitative measurement of cardiovascular performance in zebrafish embryos and larvae. BMC Biotechnol. 2007, 7, 40. [CrossRef] [PubMed]

19. Vedula, V.; Lee, J.; Xu, H.; Kuo, C.; Hsiai, T.K.; Marsden, A.L. A method to quantify mechanobiologic forces during zebrafish cardiac development using 4-D light sheet imaging and computational modeling. PLoS Comput. Biol. 2017, 13, e1005828. [CrossRef] [PubMed]

20. Lee, J.; Vedula, V.; Baek, K.I.; Chen, J.; Hsu, J.J.; Ding, Y.; Chang, C.C.; Kang, H.; Small, A.; Fei, P.; et al. Spatial and temporal variations in hemodynamic forces initiate cardiac trabeculation. JCI Insight $2018,3$. [CrossRef]

21. Liu, J.; Bressan, M.; Hassel, D.; Huisken, J.; Staudt, D.; Kikuchi, K.; Poss, K.D.; Mikawa, T.; Stainier, Y. A dual role for ErbB2 signaling in cardiac trabeculation. Development 2010, 137, 3867-3875. [CrossRef] [PubMed]

22. Reckova, M.; Rosengarten, C.; deAlmeida, A.; Stanley, C.P.; Wessels, A.; Gourdie, R.G.; Thompson, R.P.; Sedmera, D. Hemodynamics is a key epigenetic factor in development of the cardiac conduction system. Circ. Res. 2003, 93, 77. [CrossRef] 
23. Liebling, M.; Forouhar, A.S.; Wolleschensky, R.; Zimmermann, B.; Ankerhold, R.; Fraser, S.E.; Gharib, M.; Dickinson, M.E. Rapid three dimensional imaging and analysis of the beating embryonic heart reveals functional changes during development. Dev. Dyn. 2006, 235, 2940-2948. [CrossRef] [PubMed]

24. Chiu, J.J.; Chien, S. Effects of disturbed flow on vascular endothelium: Pathophysiological basis and clinical perspectives. Physiol. Rev. 2011, 91, 327-87. [CrossRef]

25. Morris, P.D.; Narracott, A.; von Tengg-Kobligk, H.; Silva Soto, D.A.; Hsiao, S.; Lungu, A.; Evans, P.; Bressloff, N.W.; Lawford, P.V.; Hose, D.R.; et al. Computational fluid dynamics modelling in cardiovascular medicine. Heart 2016, 102, 18-28. [CrossRef] [PubMed]

26. Jahnsen, E.D.; Trindade, A.; Zaun, H.C.; Lehoux, S.; Duarte, A.; Jones, E. Notch1 Is Pan-Endothelial at the Onset of Flow and Regulated by Flow. PLoS ONE 2015, 10, e0122622. [CrossRef]

27. Van der Heiden, K.; Groenendijk, B.C.; Hierck, B.P.; Hogers, B.; Koerten, H.K.; Mommaas, A.M.; Gittenberger-de Groot, A.C.; Poelmann, R.E. Monocilia on chicken embryonic endocardium in low shear stress areas. Dev. Dyn. 2006, 235, 19-28. [CrossRef]

28. Egorova, A.D.; Khedoe, P.P.; Goumans, M.J.; Yoder, B.K.; Nauli, S.M.; ten Dijke, P.; Poelmann, R.E.; Hierck, B.P. Lack of Primary Cilia Primes Shear-Induced Endothelial-to-Mesenchymal TransitionNovelty and Significance. Circ. Res. 2011, 108, 1093-1101. [CrossRef]

29. Haack, T.; Abdelilah-Seyfried, S. The force within: Endocardial development, mechanotransduction and signalling during cardiac morphogenesis. Development 2016, 143, 373-386. [CrossRef]

30. Koefoed, K.; Veland, I.R.; Pedersen, L.B.; Larsen, L.A.; Christensen, S.T. Cilia and coordination of signaling networks during heart development. Organogenesis 2014, 10, 108-125. [CrossRef]

31. Midgett, M.; Chivukula, V.K.; Dorn, C.; Wallace, S.; Rugonyi, S. Blood flow through the embryonic heart outflow tract during cardiac looping in HH13-HH18 chicken embryos. J. R. Soc. Interface 2015, 12. [CrossRef] [PubMed]

32. DeGroff, C.G.; Thornburg, B.L.; Pentecost, J.O.; Thornburg, K.L.; Gharib, M.; Sahn, D.J.; Baptista, A. Flow in the early embryonic human heart. Pediatr. Cardiol. 2003, 24, 375-380. [CrossRef] [PubMed]

33. Taber, L.; Zhang, J.; Perucchio, R. Computational model for the transition from peristalsis to pulsatile flow in the embryonic heart tube. J. Biomech. Eng. 2007, 129, 441-449. [CrossRef] [PubMed]

34. Liu, A.; Rugonyi, S.; Pentecost, J.O.; Thornburg, K.L. Finite element modeling of blood flow-induced mechanical forces in the outflow tract of chick embryonic hearts. Comput. Struct. 2007, 85, 11-14. [CrossRef]

35. Santhanakrishnan, A.; Nhi, N.; Cox, J.; Miller, L.A. Flow within models of the vertebrate embryonic heart. J. Theor. Biol. 2009, 259, 449-461. [CrossRef] [PubMed]

36. Miller, L. Fluid Dynamics of Ventricular Filling in the Embryonic Heart. Cell Biochem. Biophys. 2011, 61, 33-45. [CrossRef] [PubMed]

37. Buskohl, P.; Jenkins, J.; Butcher, J. Computational simulation of hemodynamic-driven growth and remodeling of embryonic atrioventricular valves. Biomech. Model. Mechanobiol. 2012, 11, 1205-1217. [CrossRef]

38. Lee, J.; Moghadam, M.E.; Kung, E.; Cao, H.; Beebe, T.; Miller, Y.; Roman, B.L.; Lien, C.L.; Chi, N.C.; Marsden, A.L.; et al. Moving Domain Computational Fluid Dynamics to Interface with an Embryonic Model of Cardiac Morphogenesis. PLoS ONE, 2013, 8, e72924. [CrossRef]

39. Battista, N.A.; Lane, A.N.; Miller, L.A. On the dynamic suction pumping of blood cells in tubular hearts. In Women in Mathematical Biology: Research Collaboration; Layton, A., Miller, L.A., Eds.; Springer: New York, NY, USA, 2017; Chapter 11, pp. 211-231.

40. Battista, N.A.; Lane, A.N.; Liu, J.; Miller, L.A. Fluid Dynamics of Heart Development: Effects of Trabeculae and Hematocrit. Math. Med. Biol. 2017, in press. [CrossRef]

41. Battista, N.A.; Miller, L.A. Bifurcations in valveless pumping techniques from a coupled fluid-structureelectrophysiology model of heart development. BIOMATH 2017, 6, 1711297. [CrossRef]

42. Vennemann, P.; Kiger, K.T.; Lindken, R.; Groenendijk, B.C.; Stekelenburg-de Vos, S.; ten Hagen, T.L.; Ursem, N.T.; Poelmann, R.E.; Westerweel, J.; Hierck, B.P. In vivo micro particle image velocimetry measurements of blood-plasma in the embryonic avian heart. J. Biomech. 2006, 39, 1191-1200. [CrossRef] [PubMed]

43. Jackson, B.; Evangelisa, D.J.; Ray, D.D.; Hedrick, T.L. 3D for the people: Multi-camera motion capture in the field with consumer-grade cameras and open source software. Biol. Open 2016, 5, 1334-1342. [CrossRef] [PubMed] 
44. Mohammed, M.; Roubaie, S.; Jahnsen, E.; Jones, E. Drawing First Blood: Measuring Avian Embryonic Blood Viscosity. SURE Post. Present. 2011, 61, 33-45.

45. McGaw, I.J.; Reiber, C.L. Cardiovascular system of the blue crab Callinectes sapidus. J. Morphol. 2002, 251, 1-21. [CrossRef] [PubMed]

46. Wilbur, K.; Yonge, C. Physiology of Mollusca; Number v. 2; Elsevier Science: Amsterdam, The Netherlands, 2013.

47. Childs, H.; Brugger, E.; Whitlock, B.; Meredith, J.; Ahern, S.; Pugmire, D.; Biagas, K.; Miller, M.; Harrison, C.; Weber, G.H.; et al. VisIt: An End-User Tool For Visualizing and Analyzing Very Large Data. In High Performance Visualization-Enabling Extreme-Scale Scientific Insight; CRC Press: Boca Raton, FL, USA, 2012; pp. 357-372.

48. Shen, C.; Floryan, J.M. Low Reynolds number flow over cavities. Phys. Fluids 1985, 28, 3191. [CrossRef]

49. Mizushima, J.; Okamoto, H.; Yamaguchi, H. Stability of flow in a channel with a suddenly expanded part. Phys. Fluids 1996, 8, 2933-2942. [CrossRef]

50. Sedmera, D. Evolutionary and Developmental Views on Ventricular Myocardium; Technical Report; European Society of Cardiology: Sophia Antipolis, Nice, France, 2010.

51. Burggren, W.W. Cardiac design in lower vertebrates: What can phylogeny reveal about ontogeny? Experientia 1988, 44, 919-930. [CrossRef]

52. Burggren, W.W.; Warburton, S.J. Patterns of form and function in developing hearts: Contributions from non-mammalian vertebrates. Cardioscience 1994, 5, 183-191.

53. Collis, L.P.; Sun, Y.; Hill, R.B. Length-dependent deactivation of ventricular trabeculae in the bivalve, Spisula solidissima. J. Comp. Physiol. B 2006, 176, 371-385. [CrossRef] [PubMed]

54. Hu, N.; Sedmera, D.; Yost, H.J.; Clark, E.B. Structure and function of the developing zebrafish heart. Anat. Rec. 2000, 260, 148-157. [CrossRef]

55. Kodirov, S.A. The neuronal control of cardiac functions in Molluscs. Compar. Biochem. Physiol. Part A Mol. Integr. Physiol. 2011, 160, 102-116. [CrossRef]

56. Tucker, D.C.; Snider, C.; Woods, W.T., Jr. Pacemaker development in embryonic rat heart cultured in oculo. Pediatr. Res. 1988, 23, 637-642. [CrossRef] [PubMed]

57. Chapman, W. The effect of the heart-beat upon the development of the vascular system in the chick. Am. J. Anat. 1918, 23, 175-203. [CrossRef]

58. Weinbaum, S.; Zhang, X.; Han, Y.; Vink, H.; Cowin, S. Mechanotransduction and flow across the endothelial glycoalyx. Prod. Natl. Acad. Sci. USA 2003, 100, 7988-7995. [CrossRef] [PubMed]

59. Paluch, E.K.; Nelson, C.M.; Biais, N.; Fabry, B.; Moeller, J.; Pruitt, B.L.; Wollnik, C.; Kudryasheva, G.; Rehfeldt, F.; Federle, W. Mechanotransduction: Use the force(s). BMC Biol. 2015, 13, 47. [CrossRef] [PubMed]

60. Chen, L.; Wei, S.; Chiu, J. Mechanical regulation of epigenetics in vascular biology and pathobiology. J. Cell. Mol. Med. 2014, 11, 437-448. [CrossRef] [PubMed]

61. Olesen, S.P.; Clapham, D.E.; Davies, P.F. Hemodynamic shear-stress activates a K+ current in vascular endothelial cells. Nature 1988, 331, 168-170. [CrossRef]

62. Davies, P.F.; Remuzzi, A.; Gordon, E.J.; Dewey, C.F.; Gimbrone, M.A. Turbulent fluid shear-stress induces vascular endothelial-cell turnover in vitro. Proc. Natl. Acad. Sci. USA 1986, 83, 2114-2117. [CrossRef]

63. Peskin, C. The immersed boundary method. Acta Numer. 2002, 11, 479-517. [CrossRef]

64. Jung, E.; Peskin, C. 2-D simulations of valveless pumping using immersed boundary methods. SIAM J. Sci. Comput. 2001, 23, 19-45. [CrossRef]

65. Hershlag, G.; Miller, L.A. Reynolds number limits for jet propulsion: A numerical study of simplified jellyfish. J. Theor. Biol. 2011, 285, 84-95. [CrossRef] [PubMed]

66. Bhalla, A.; Griffith, B.; Patankar, N. A forced damped oscillation framework for undulatory swimming provides new insights into how propulsion arises in active and passive swimming. PLoS Comput. Biol. 2013, 9, e1003097. [CrossRef] [PubMed]

67. Tytell, E.; Hsu, C.; Williams, T.; Cohen, A.; Fauci, L. Interactions between internal forces, body stiffness, and fluid environment in a neuromechanical model of lamprey swimming. Proc. Natl. Acad. Sci. USA 2010, 107, 19832-19837. [CrossRef] [PubMed]

68. Battista, N.A.; Strickland, W.C.; Miller, L.A. IB2d: A Python and MATLAB implementation of the immersed boundary method. Bioinspir. Biomim. 2017, 12, e036003. [CrossRef] [PubMed]

69. Battista, N.A.; Strickland, W.C.; Barrett, A.; Miller, L.A. IB2d Reloaded: A more powerful Python and MATLAB implementation of the immersed boundary method. Math. Method. Appl. Sci. 2018, 41, 8455-8480. [CrossRef] 
70. Griffith, B. An Adaptive and Distributed-Memory Parallel Implementation of The Immersed Boundary (IB) Method. Available online: https:/ /github.com/IBAMR/IBAMR (accessed on 14 January 2018).

71. Griffith, B.; Hornung, R.; McQueen, D.; Peskin, C. An adaptive, formally second order accurate version of the immersed boundary method. J. Comput. Phys. 2007, 223, 10-49. [CrossRef]

72. Berger, M.J.; Oliger, J. Adaptive mesh refinement for hyperbolic partial-differential equations. J. Comput. Phys. 1984, 53, 484-512. [CrossRef]

73. Berger, M.J.; Colella, P. Local adaptive mesh refinement for shock hydrodynamics. J. Comput. Phys. 1989, 82, 64-84. [CrossRef]

74. Williams, H.A.; Fauci, L.J.; Gaver, D.P., III. Evaluation of interfacial fluid dynamical stresses using the immersed boundary method. Disc. Contin. Dyn. Syst. Ser. B 2012, 11, 519-540. [CrossRef]

(C) 2019 by the authors. Licensee MDPI, Basel, Switzerland. This article is an open access article distributed under the terms and conditions of the Creative Commons Attribution (CC BY) license (http:/ / creativecommons.org/licenses/by/4.0/). 